\title{
Bambusoideae in India: An Updated Enumeration
}

\author{
Pushpa Kumari \\ Botanical Survey of India, Central National Herbarium, \\ P.O.: Botanic Garden, Howrah - 711103 (India) \\ Corresponding Author: pushparbg@gmail.com Mobile: +919434207721
}

\section{Manuscript Details}

Manuscript Submitted : 10/03/2019

Manuscript Revised : :12/03/2019

Manuscript Accepted : 13/03/2019

Manuscript Published : 15/03/2019

\section{$\underline{\text { Available On }}$}

https://plantaescientia.website/ojs

\section{Cite This Article As}

Pushpa Kumari (2019). Bambusoideae in India: An updated enumeration, Pla. Sci. 2019; Vol. 01 Iss. 06: 99-117. DOI:https://doi.org/10.32439/ps.vli06.99$\underline{117}$

\section{$\underline{\text { Copyright }}$}

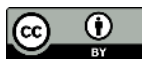

(c) The Author (s). 2019. Open Access This article is distributed under the terms of the Creative Commons Attribution 4.0 International License http://creativecommons.org/licenses/by/4.0/

\section{$\underline{\text { Indexed In }}$}

Crossref, Index Copernicus International (ICI), Directory of Research Journal Indexing (DRJI), Scientific Indexing Services (SIS), CiteFactor

\begin{abstract}
Bamboo a giant, fast-growing, wood like grass appears to be the most successful and diverse conspicuous group of plants belonging to the sub family Bambusoideae of Poaceae. Bamboos have strong adaptability and are distributed widely in varied habitats. They are one of the earth's oldest and most precious plant materials and have benefited human societies since time immemorial. They form an important group of plants that play a vital role in the economy and life of the people in many Asian, African and American countries. Bamboos play a key role in the rural economy of India. Due to the special physical characteristics attributed to bamboo, it has a glorious past and a promising future as part of the solution to $21^{\text {st }}$ century challenges. Globally, bamboo is being targeted for livelihood development and alleviation of both environment and social problems in such a way that it can rightly be called the plant of the century.

Taxonomically, bamboos are considered as one of the most difficult group of plants to identify. Despite of immense resources, its importance and species specific uses, taxonomy of Indian Bamboos is still incomplete. Many of the Indian species lack full description and are either partially known or misidentified due to lack of subject expertise. Different workers treat species differently which has resulted into ambiguous report of generic and infrageneric taxa. An updated account of the bamboos found or reported from India is presented here after an extensive taxonomic study of these valuable natural resources of our country.
\end{abstract}

Keywords: Bamboo, Bambusoideae, Enumeration, India. 


\section{INTRODUCTION}

The very first mention of bamboo in literature dates back to $300 \mathrm{BC}$ in a letter from Alexander the Great to Aristotle. The first pre-Linnaean scientific writer on Indian bamboos was van Rheede, the then Dutch Governor of Malabar, who described one species namely 'Ily' in his Hortus Malabaricus Vol. I, presently known as Bambusa bambos and two species viz., 'Beesha' and 'Nola-ily' in Vol. V which are presently known as Ochlandra scriptoria and O. travancorica respectively. However, Linnaeus in his $1^{\text {st }}$ edition of Species Plantarum mentioned only one species as Arundo bambos, now referable to Bambusa bambos. In 1789 the first bamboo genus viz., "Bambos" was scientifically described by Retzius on the basis of a single species Bambos arundinacea, now called Bambusa bambos. In the same year the generic name "Bambusa" was proposed by Schreber with the generic name "Bambos" as its synonym. Seven species viz., Bambusa bambos, B. stricta, B. tulda, B. balcooa, B. baccifera, B. spinosa and B. nana were enumerated in 'Hortus Benghalensis' by William Roxburgh (1814). It was in 1815 for the first time, that bamboos were treated in separate class in grass systematics by Charles Kunth, where he placed the bamboos in "Gramina Bambusacea" Class X of his treatment. The work of Christian Nees von Esenbeck (1835) was the first taxonomic monograph for bamboos. Russian botanist Franz Ruprecht (1839) published a monograph on bamboos where he placed 67 species of woody bamboos, including 18 from IndoMalayan region, in two groups. The excellent monograph by Colonel Munro (1868) covering the bamboos of the world, was more complete and introduced a new section Bacciferae. He enumerated 219 species including 70 species from IndoMalayan region under 20 genera, placed in three sub-tribes. In his Forest Flora of Burma, Kurz (1877) described 30 species. In 1881, George Bentham in his classic paper 'Notes on Gramineae' proposed four subtribes of bamboos adopting the concept of Nees (1835) and Munro (1868) with slight modifications in names and ranks.

The monumental work of James S. Gamble (1896) was the most complete monograph on Indian bamboos. It enumerated 115 species of 15 genera under 4 subtribes proposed by Bentham (1881), occurring in India, Pakistan, Bangladesh, Myanmar, Malaya and Sri Lanka. Brandis (1906) in his Indian Trees, listed 14 genera and 108 species. This was followed by monographic work of Camus (1913) which included the bamboos of India and China. Blatter (1929) presented a systematic analysis and arrangement of Indian bamboos. He added 24 new species from India since Gamble's (1896) publication. Majumdar's enumeration of Indian bamboos was published in Karthik. et al., (1989) where he treated several species under synonyms and described 91 species under 18 genera including 8 new species and 1 new genus. Besides new taxa, he proposed several combinations. Thirty Seven Bamboos Growing in India by Bennet $\&$ Gaur (1990) aided with the coloured illustrations of the bamboos growing in FRI Dehradun arboretum is a useful attempt for field identification.

The first 'monograph' on bamboos of India after independence by D.N. Tiwari (1992), contains description, distribution, flowering records and uses of 129 species under 23 genera, including indigenous as well as cultivated species. The work of Negi and Naithani (1994) provides useful information of important bamboos of India in a concise form. The recent monographic work on Indian bamboos is that of Seethalakshmi \& Muktesh Kumar (1998), which is another compilation work based on literature and provides detail information on 128 species belonging to 18 genera.

In the taxonomic treatment of bamboos the system of classification adapted by Munro which was mainly based on the flowering structures, was followed by Gamble with some modifications. But since the flowering in bamboos is very rare and irregular and most of them die after flowering, making it difficult to collect a bamboo species in flower along with the important vegetative parts such as culmsheaths and leaves, the vegetative structures are being given more importance for the identification and classification by subsequent and recent workers. As a result, there have been more and often changes in Generic concept among the taxonomists. The larger heterogeneous groups have been split into many smaller genera, for example almost all species treated under genus Arundinaria have been transferred under Indocalamus, Himalayacalamus, Drepanostachyum, Borinda, Sinarundinaria, Yushania, Fargesia, Neomicrocalamus, Racemobambos, Thamnocalamus, Chimonocalamus, Sarocalamus etc. and the genus Arundinaria at present is having only 3 species worldwide (Vorontsova et al., 2016). Similarly the genera Cephalostachyum, Pseudostachyum and Teinostachyum were suggested to be merged with Schizostachyum by Holttum (1958) who proposed a classification system based on the structure of ovary and divided all the genera into four groups. The concept was agreed by Clayton and Renvoize (1989) and followed by subsequent workers Majumdar (1989), Tiwari (1992), Shukla (1996) and Seethalakshmi \& Kumar (1998), but Dransfield \& Widjaja (1995) was clear to keep all these genera separate and this idea was followed by Stapleton (2000) and most of the Asian workers. While describing Stapletonia a new genus authors are also of the opinion that these genera should be treated separately (Dash et al., 2009).

Subfamily Bambusoideae includes 1642 species including 1521 herbaceous species, worldwide (Vorontsova et al., 2016). During last 125 years after the monumental work of Gamble (1896), 3 new genera and more than 35 species new to 
science have been discovered and described from India, mostly North eastern region, Western Ghats and Andaman and Nicobar islands.

\section{ENUMERATION}

Present paper based on extensive taxonomic research on this group of plants for more than fifteen years from 2002 to 2018, enumerates total of 148 species and 6 varieties under 33 genera from India. It includes indigenous, widely cultivated and naturalized and the exotic species used particularly for ornamental purposes in landscaping gardens. It also enlists the species mentioned as occurring in India in previous works but found of doubtful occurrence in the present work. 15 genera are under Aruninariae and 18 are under Bambusiae [Table:1]. However, here all the genera are cited alphabetically with current accepted name, followed by the basionym and other important synonynms. Type(s) of the name, followed by the vernacular names and the distribution of species in Indian states and in other countries are provided for each species.

AMPELOCALAMUS S.L. Chen, T.H.Wen \& G.Y.Sheng, Acta Phytotax. Sin. 19:332. 1981.

Type: Ampelocalamus actinotrichus (Merrill \& Chun) S.L.Chen, T.H.Wen \& G.Y. Sheng

Ampelocalamus patellaris (Gamble) Stapleton, Edinburgh J. Bot. 51(3): 321. 1994. Dendrocatamus patellaris Gamble, Ann. Roy. Bot. Gard. Calcutta 7: 86-87. t. 75. 1896. Patellocalamus patellaris (Gamble) W.T.Lin, J. S. China Agric. Univ. 10(2): 46. 1989. Sinocalamus patellaris (Gamble) T.Q.Nguyen, Bot. Zurn. (Moscow \& Leningrad) 74:1662. 1989. Chimonobambusa jainiana C.R.Das \& D.C.Pal, J. Econ. Taxon. Bot. 4(3): 1023. 1983. Drepanostachyum jainianum (C.R.Das \& D.C.Pal) R.B.Majumdar, Bull. Bot. Surv. India 25(1-4): 235. 1983. Sinarundinaria jainiana (C.R.Das \& D.C.Pal) H.B.Naithani, Indian Forester: 116(12): 990. 1990.

Type: Sikkim, Jungat, alt. 4000 ft., November, 1881, Gamble 10045 (K). LT designated by Stapleton in Edinburgh J. Bot. 5l: 321. 1994.

Vern.: Pagjiok, Pagjiok-pao (Lepch); Footoong (Mikir). Distribution: INDIA: Arunachal Pradesh, Assam, Nagaland, Sikkim, Uttarakhand, West Bengal. [Bhutan, China, Laos, Myanmar, Nepal].

BAMBUSA Schreb., Gen. Pl, ed. 8[a] 236.1789, nom. cons. Type: Bambusa bambos (L.) Voss.

Bambusa alemtemshii H.B.Naithani, Indian Forester 133(11): 1575. 2007.

Type: India: Nagaland, Mokokchung District, Longsa village, December 5, 2004, H.B.Naithani 4338 (DD).

Vern.: Alulem (Ao).
Distribution: Assam, Nagaland. Endemic.

Bambusa balcooa Roxb., Fl. Ind. 2: 196. 1832.

Lectotype: Roxb. Icones 1402 (K). LT designated by Stapleton in Edinburgh J. Bot 5l(1): 12. 1994.

Vern.: Baluka (Ass.); Balku (Beng.); Wamnah, Beru (Garo); Barak (Tripura).

Distribution: Arunachal Pradesh, Assam, Bihar, Kerala, Manipur, Meghalaya, Nagaland, Orissa, Tripura, Uttarakhand, Uttar Pradesh, West Bengal. [Bangladesh, China].

Bambusa bambos (L.) Voss, Vilm. Blumengartn. ed. 3. 1: 1189. 1895. Arundo bambos L. Sp. Pl. 1:81. 1753. Bambusa arundinacea (Retz.) Willd. Sp. Pl. ed. 4 [Willdenow] 2: 245. 1799. Bambos arundinacea Retz., Observ. Bot. (Retzius) 5: 24. 1788. Bambusa bambos (L.) Voss var. gigantea Bennet \& R.C.Gaur, Thirty Seven Bamboos Growing in India. 21. 1990. Lectotype: "Ily" in Rheede, Hort. Malab. 1: 25. t. 16. 1678. Lectotype designated by Judziewiez in Gorts-van Rijn (ed.), Fl. Guianas, see. A. 8: 50. 1990.

Vern.: Kotoba (Ass.); Baroowa, Behor, Ketuasi, Ketwa (Beng.); Saneibi (Manipuri); Daba, Kating (Orrisa); Mulkas veduru, Mullu veduru (Telugu).

Distribution: Andhra Pradesh, Assam, Chhattisgarh, Goa, Gujarat, Himachal Pradesh, Karnataka, Kerala, Madhya Pradesh, Maharashtra, Manipur, Meghalaya, Mizoram, Rajasthan, Tamil Nadu, West Bengal. [Bangladesh, China, Myanmar, Pakistan, Sri Lanka].

Bambusa burmanica Gamble, Ann. Roy. Bot. Gard. Calcutta 7: 35-36. t. 33. 1896.

Type: Burma: Katha District, dry slopes, J.W.Oliver s.n. (K). Vern.: Thaikawa (Ass.).

Distribution: Arunachal Pradesh, Assam, Mizoram, Uttarakhand, West Bengal. [China, Malayasia, Myanmar].

Bambusa cacharensis R.B.Majumdar, Bull. Bot. Surv. India 25(1-4): 237. f. 1. 1983.

Type: India: Cachar, Lakhimpur, Mar Bastee, R.B.Majumdar 74265A (CAL).

Vern.: Betua (Beng.); Moirang-wa (Manipuri).

Distribution: Assam, Manipur, Meghalaya, Tripura, West Bengal. Endemic.

Bambusa dampaeana H.B.Naithani, Gabriyal \& Bisht, in Indian Forester 136(7): 991-992. 2010.

Type: India: Mizoram, Terei, on way to Dampa, 11.12.2009, H.B.Naithani 4344 (Holo DD).

Distribution: Mizoram. Endemic.

Bambusa garuchokua Barooah \& Borthakur, in Indian J. Forestry 24(4): 506-508. 2001. 
TABLE 1: List of Taxa Reported from India under Subfamily Bambusoideae (Poaceae)



Type: India: Assam, Lakhimpur, Barpathar No.1, 7.7.1996, C.Barooah 2505 (Holo ASSAM); Bor Azara, Nalbari, Assam, India, 8.3.1997, C.Barooah 2532 (Para CAL); Chapor, Dhubri, Assam, India, 8.3.1998, C.Barooah 2567 (Para Gauhati University Herb., Guwahati).

Distribution: Assam. Endemic.

Bambusa jaintiana R.B.Majumdar in Karthik. et al., Fl. Ind. Enumerat.- Monocot. 274. 1989. Bambusa alamii Stapleton, Edinb. J. Bot. 51(1): 10. Fig 3. 1994. Bambusa assamica Barooah $\&$ Borthakur, in Indian J. Forestry 24(4): 503-505. 2001.

Type: India: Shillong, G.K.Deka 31765 (CAL).

Vern.: U-shken (Khasi); Gasui, Gasai (Rongmai); Mirtianga (Beng.).
Distribution: Arunachal Pradesh, Assam, Meghalaya Manipur, Nagaland, West Bengal. [Bangladesh, Bhutan, Myanmar, Nepal].

Bambusa khasiana Munro, Trans. Linn. Soc. London 26(1): 97. 1868.

Syntypes: India: Khasia, alt. 2000-4000 ft., Churra, Hooker no. 1097 (K); Jasper Hill Mahadeb Hooker 496 (K).

Distribution: Assam, Manipur, Meghalaya. [Myanmar].

Bambusa majumdarii P.Kumari \& P.Singh, in Kew Bull. 64(3):565. 2009.

Type: India: Meghalaya, Near Tura, Garo Hills, 14.9.2005, P.Kumari \& P.Singh 34696 (Holo CAL; Iso ASSAM). Vern.: Wa-nari (Garo). 
Distribution: Meghalaya. Endemic.

Bambusa manipureana H.B. Naithani, Indian Forester 136(2): 262-263. 2010.

Type: India: Manipur, Chandel district, $10 \mathrm{~km}$ before Moreh, 11.10.2008, H.B.Naithani 5011 (Holo DD).

Vern.: Lawom, Uying (Chote Tribe); Uma (Manipuri); Govam, Gosing (Kuki).

Distribution: Manipur. Endemic.

Bambusa mohanramii P.Kumari \& P.Singh, in Kew Bull. 64(3):567. 2009.

Type: India: Meghalaya, Jaintia Hills, Khleiriaht, 9.4.2004,

P.Kumari 34608 (Holo CAL; Iso ASSAM).

Vern.: Seij (Jaintia).

Distribution: Meghalaya. Endemic.

Bambusa multiplex (Lour.) Raeusch. ex Schult. \& Schult.f., Syst. Veg., ed. 15 bis [Roemer \& Schultes] 7(2): 1350. 1830. Arundo multiplex Lour., Fl. Cochinch. 1: 58. 1790. Bambusa nana Roxb., Fl. Ind. (Roxburgh) 2: 199. 1832. Ludolfia glaucescens Willd., Ges. Naturf. Freunde Berlin Mag. Neuesten Entdeck. Gesammten Naturk. 2: 320. 1808. Bambusa glaucescens (Willd.) Siebold ex Munro. Trans. Linn. Soc. London 26(1): 89. 1868. nom. invalid, pro. syn.

Type: No type specimen exists (Stapleton 1994).

Distribution: Assam, Bihar, Meghalaya, West Bengal. [Bangladesh, China, Myanmar, Sri Lanka]. Probably naturalized in addition to being cultivated.

Bambusa nairiana P.Kumari \& P.Singh, in Bull. Burv. India 50:13. 2008.

Type: India: Meghalaya, Garo Hills, Near Nokrek, 14.9.2005,

P.Kumari \& P.Singh 34698 (Holo CAL; Iso ASSAM).

Vern.: Wa-sim (Garo).

Distribution: Meghalaya. Endemic.

Bambusa nutans Wall ex Munro, Trans. Linn. Soc. London 26(1): 92.1868.

Lectotype: Nepal: Kathmandu, Naga-Arjun [Nagarjun, $27^{\circ}$ 44'N 857l'E]: 1821; Numer. List [Wallich] no. 5031(K-W). LT designated by Stapleton in Edinburgh J. Bot. 5l: 17. 1994. Vern.: Deobans, Jotia-makal, Bidhuli, Mukial (Ass.); Seringjai (Khasi); Wa-malang (Kuki); Mahi bans, Mahlu, Mallo (Lepcha); Peechli, Kali (Trpura).

Distribution: Arunachal Pradesh, Assam, Bihar, Himachal Pradesh, Madhya Pradesh, Orissa, Sikkim, Tripura, Uttar Pradesh, Uttarakhand, West Bengal. [Bangladesh].

Bambusa oliveriana Gamble, Ann. Roy. Bot. Gard. Calcutta 7: 130. t. 116. 1896. Bambusa mizorameana H.B. Naithani, in Indian Forester 135(9): 1291-1292. 2009.

Syntypes: Burma: 30 miles north from Mandalay, alt. 10002000 ft., 1893-94, J.W.Oliver s.n.; Ruby Mines Hills (K); C.S.Rogers s.n. (CAL).
Vern.: Talan (Mizo); Khokwa (Manipuri); Khuak (Kabui, Rongmei); Rokhu (Tangkhul).

Distribution: Manipur, Sikkim, Uttarakhand, West Bengal. [Myanmar]. Naturalized.

Bambusa pallida Munro, Trans. Linn. Soc. London 26(1): 97. 1868. Bambusa barpatharica Borthakur \& Barooah, Indian J. Forestry 24(4): 505-506. 2001. Bambusa mompaeana H.B. Naithani, Indian Forester 141(5): 587-589. 2015. Bambusa rangaensis Borthakur \& Barooah, Indian J. Forestry 24(4): 508-509. 2001.

Syntypes: India: Bengalia, Pundua \& Sylhet (cult.). Cachar, Khasia, Joowye. alt. 3500 ft. Hooker \& Thomson 602, 607 (K). Vern.: Bijli, Jowa, Makal, Walkthai (Ass.); Bakhal, Burwal (Cachar); Seskien, Skhen, Tneng, Usken (Khasi); Pashipo, Pshi, Pushee (Lepcha); Loto (Mikir); Tesero, Watoi (Naga); Makal (Tripura).

Distribution: Arunachal Pradesh, Assam, Meghalaya, Mizoram, Nagaland, Sikkim, Tripura, Uttar Pradesh, West Bengal. [Bangladesh, Bhutan, China, Myanmar].

Bambusa polymorpha Munro, Trans. Linn. Soc. London 26(1): 98. 1868.

Syntypes: Burma: Martaban, Pegu. ("Kijathounwa") Brandis no. 1; Rangoon, M'Clelland s.n. (K).

Vern.: Jama betwa, Betwa, Bethua-bah (Ass.); Betua, Jaibaroowa, Jama (Beng.); Narangi bhas (Madhya Pradesh); Bari (Tripura).

Distribution: Arunachal Pradesh, Assam, Karnataka, Kerala, Madhya Pradesh, Manipur, Meghalaya, Tamil Nadu, Tripura, West Bengal. [Bangladesh, China, Myanmar].

Bambusa pseudopallida R.B.Majumdar in Karthik. et al., Fl. Ind. Enumerat.- Monocot. 275. 1989.

Type: India: Umtaph on Dawki-Jarain Road, N.P.Balakrishnan 42700 (CAL).

Distribution: Assam, Meghalaya. Endemic.

Bambusa teres Buch.-Ham. ex Munro, Trans. Linn. Soc. London 26(1): 95. 1868.

Syntypes: India: Bengal, Gongachora; Buchanan-Hamilton 881, Griffith s.n. Assam; Jenkins s.n. (K).

Vern.: Bhaluki-makal (Ass.); Paora (Tripura); Saneibi (Manipuri); Ankuang Mizo); Vakila (Bru/ Riang).

Distribution: Arunachal Pradesh, Assam, Chhatisgarh, Madhya Pradesh, Meghalaya, Nagaland, Tripura, West Bengal. [Bangladesh, Bhutan, China, Myanmar, Nepal].

Bambusa tulda Roxb., Fl. Ind. (Roxburgh) 2: 193. 1832. Lectotype: Roxb. Icones 1403 (K). ). LT designated by Stapleton in Edinburg J. Bot. 5 l (1): 19. 1994.

Vern.: Wamuna, Wagi, Nal-bans (Ass.); Tulda, Jowa (Beng.); Kiranti, Matela (Duars); Wati (Garo); Bijuli, Jati, Jao, Ghora (Kamrup); Mirtinga (Tripura). 
Distribution: Andhra Pradesh, Arunachal Pradesh, Assam, Bihar, Jharkhand, Karnataka, Meghalaya, Mizoram, Nagaland, Orissa, Tripura, Uttar Pradesh, Uttarakhand, West Bengal. [Bangladesh, Bhutan, China, Myanmar, Nepal].

Bambusa tulda Roxb. var. gamblei P.Kumari \& P.Singh, Bamboos of Meghalaya p. 58. 2014. Botanical Survey of India, Kolkata, India.

Type: India: Meghalaya, Garo Hills, Near Nokrek, 14.9.2005,

P.Kumari \& P.Singh 346700 (Holo CAL).

Distribution: Meghalaya. Endemic.

Bambusa vulgaris Schrad, in J.C.Wendl. Coll. Pl, 2: 26, t. 47. 1810.

Type: India: cultivated (BM (possible)).

Vern.: Bakal (Beng. and Manipuri); Ranai-shilot (Khasi); Vairua (Mizo); Bachiabas (Chakma); Uasur (Bru/ Riang); Sunderkania bans (Orissa).

Distribution: Arunachal Pradesh, Assam, Bihar, Chhatisgarh, Goa, Kerala, Madhya Pradesh, Maharashtra, Manipur, Meghalaya, Orissa, Rajasthan, West Bengal. [Bangladesh, China, Sri Lanka].

Bambusa vulgaris Schrad. ex Wendl. var. vittata Riviere \& C.Riviere, Bambous 191. 1878. Bambusa vulgaris Schrad. var. striata (Lodd. ex Lindl.) Gamble, Ann. Roy. Bot. Gard. Calcutta 7: 44. 1896. Bambusa striata Lodd. ex Lindl., Penny Cyclop. 3: 357. 1835.

Type: In China, in Anglia saepe culta, loddiges.

Vern.: Basini bans (Beng.); Sundrogai (Orissa); Yellow bamboo (Eng.).

Distribution: Assam, Bihar, Meghalaya, Manipur, West Bengal. [China].

CEPHALOSTACHYUM Munro, Trans. Linn. Soc. London 26: 138.1868.

Type: Cephalostachyum capitatum Munro

Cephalostachyum capitatum Munro, Trans. Linn. Soc. London 26(1): 139. 1868. Schizostachyum capitatum (Munro) R.B.Majumdar in Karthik. et al., Fl. Ind. Enumerat.Monocot. 281. 1989. non (Trin.) Rupr. 1840. Schizostachyum munroi S.Kumar \& P.Singh, J. Indian Bot. Soc. 70(1-4): 423. 1991.

Syntypes: "Hab. in In dia or montibus, Khasia, Churra, 4000 ped. s.m.: Wallich et Griffith 1078, 1392 (in Herb. Griff.); Nunklow ("Sillee et Sullea"), no. 1813; Sikkim, 4000-6000 ft., Myong Valle ("Pyong"), Hooker e Thomson."

Vern.: Gope bans (Nepal); Payong (Lepcha); Ternap (Khasi); Nhu, Naat (Manipuri); Nagatheo (Tangkhul); Nat (Mizo).

Distribution: Arunachal Pradesh, Assam, Meghalaya, Mizoram, Nagaland, Sikkim. [Bhutan].
Cephalostachyum capitatum Munro var. decompositum Gamble, Ann. Roy. Bot. Gard. Calcutta 7: 105. 1896. Schizostachyum capitatum (Trin.) Rupr. var. decompositum (Gamble) R.B.Majumdar in Karthik. et al., Fl. Ind. Enumerat.- Monocot. 281. 1989.

Type: India: Sikkim: T.Anderson \& Kurz s.n. (K).

Distribution: Sikkim, Meghalaya. Endemic.

Cephalostachyum fuchsianum Gamble, Ann. Roy. Bot. Gard. Calcutta 7: 107. t. 94. 1896. Schizostachyum fuchsianum (Gamble) R.B.Majumdar in Karthik. et al., Fl. Ind. Enumerat.- Monocot. 281. 1989.

Type: India: Sikkim, Songchonglu, alt. 6000 ft., 1889-1892, Gammie s.n. (K).

Vern.: Palom (Lepcha); Rawneal (Mizo); Rania, Rangia (Mara), Ramrikawn (Paithe); Chama (Liangmei); Gowa (Kuki); Ngai (Tangkhul).

Distribution: Arunachal Pradesh, Manipur, Mizoram, Nagaland, Sikkim, West Bengal. [Bhutan, China, Myanmar, Nepal].

Cephalostachyum latifolium Munro, Trans. Linn. Soc. London 26(1): 140. 1868. Schizostachyum latifolium (Munro) R.B.Majumdar in Karthik., Fl. Ind. Enumerat.- Monocot. 281. 1989. non Gamble. 1896.

Lectotype: Bhutan: W.Griffith 2682 (K). LT designated by Stapleton in Edinburgh J. Bot. 51:

29. 1994.

Distribution: Arunachal Pradesh, Manipur, Nagaland, Sikkim, West Bengal. [Bhutan, Myanmar, Nepal, Yunnan].

Cephalostachyum longwanum H.B.Naithani, Indian Forester 136(3): 406-407. 2010.

Type: India: Nagaland, Mon District, Longwa Village, 8.12.2004, H.B.Naithani 4343 (Holo DD).

Vern.: Nahnyii (Konyak).

Distribution: Nagaland. Endemic.

Cephalostachyum mishimieanum H.B.Naithani, Indian Forester 140(7): 733-734. 2014

Type: India: Arunachal Pradesh, Lohit dist., way to Hayuling, $5 \mathrm{~km}$ after Udayak Pass, $1200 \mathrm{~m}, 29.4 .2012$, H.B.Naithani 5241 (Holo DD); Hunli, 1300m, Upper Dibang Valley District, 11.8.2012, H.B.Naithani 5293 (Para DD). Vern.: Kapit, Kapi (Digaru Mishimi); Apit (Edu Mishimi). Distribution: Arunachal Pradesh. Endemic.

Cephalostachyum pallidum Munro, Trans. Linn. Soc. London 26(1): 139. 1868. Schizostachyum pallidum (Munro) R.B.Majumdar in Karthik. et al., Fl. Ind. Enumerat. Monocot. 282. 1989.

Type: India: Mishmee, Birmah, Patkaye, 5000 ft., Griffith s.n. (K).

Vern.: Mayung (Manipuri). 
Distribution: Arunachal Pradesh, Manipur, Meghalaya. [Myanmar, Yunnan].

Cephalostachyum pergracile Munro, Trans. Linn. Soc. London 26(1): 14. 1868. Schizostachyum pergracile (Munro) R.B.Majumdar in Karthik. et al., Fl. Ind. Enumerat. Monocot. 282. 1989.

Syntypes: Burma: Rangoon, J.E.McClelland s.n. (K); Pegu, Brandis s.n.

Vern.: Bhala bans (Madhya Pradesh); Madang (Ass.); Wootang, Pungsang (Manipur); Latang (Naga); Luphul (Moyan Naga); Gulhang, Goekhang (Kuki); Mau-dang (Mizo); Dangi (Orrisa).

Distribution: Assam, Andhra Pradesh, Arunachal Pradesh, Bihar, Madhya Pradesh, Manipur, Nagaland, Orissa, West Bengal. [China, Myanmar].

\section{CHIMONOBAMBUSA Makino, Bot. Mag. (Tokyo) 28:}

\section{1914.}

Lectotype: Chimonobambusa marmorea (Mitford) Makino (Bambusa marmorea Mitford). LT designated by McClure in Taxon 6(7): 201-202. 1957.

Chimonobambusa callosa (Munro) Nakai, J. Arnold Arbor. 6(3): 151. 1925. Arundinaria callosa Munro, Trans. Linn. Soc. London 26(1): 30. 1868. Chimonocalamus callosus (Munro) J.R.Xue \& T.P.Yi, Acta Bot. Yunnan. 1(2): 84. 1979. Chimonobambusa arunachalensis Sharma \& Borthakur, Pleione 2(1): 1-2. 2008. Chimonobambusa jainii Sharma \& Borthakur, J. Econ. Taxon. Bot. 32(1): 783-785. 2008.

Lectotype: India: Meghalaya, Myrung, July 6, 1850, J.D.Hooker $\&$ Thomson 1504 (K). LT designated by C.S. Chao \& Renvoize in Kew Bull. 44(2): 366. 1989.

Vern.: Uskong, Uspar, Spar, Sypar, Spar-iong (Khasi); Laiwa (Manipuri), Ngatha, Ngth (Tangkhul); Pangki (Kabui, Rongmei).

Distribution: Arunachal Pradesh, Assam, Manipur, Meghalaya, Nagaland. [Bhutan].

Chimonobambusa quadrangularis (Franseschi) Makino, Bot. Mag. Tokyo 28: 153. 1914. Bambusa quadrangularis Franseschi, Bull. Soc. Tosc. Ortic. 5: 401. 1880.

Type: n.v.

Vern.: U-sparbah (Khasi).

Distribution: Meghalaya, Tamil Nadu, West Bengal. Probably naturalized in Mawklat, Meghalaya.

CHIMONOCALAMUS J.R.Xue \& T.P.Yi, Acta Bot. Yunnan. 1(2): 76. 1979.

Type: Chimonocalamus delicatus J.R.Xue \& T.P.Yi.

Chimonocalamus griffithianus (Munro) J.R.Xue \& T.P.Yi, Acta Bot. Yunnan.
1(2): 83-84. 1979. Arundinaria griffithiana Munro, Trans. Linn. Soc. London 26(1): 20. 1868. Chimonobambusa griffithiana (Munro) Nakai, J. Arnold Arbor. 6(3): 151. 1925. Sinarundinaria griffihiana (Munro) C.S.Chao \& Renvoize, Kew Bull. 44(2): 353. 1989.

Syntypes: India: Assam, Khasia Mountain, Moflong, Griffith Assam Dept. 39 (K); J.D.Hooker s.n. (K).

Vern.: Khnap, Uspar, Knap, Ukhnap, Usknap, U-spar-iong (Khasi \& Jaintia); Phar (Mizo); Aphoa (Mara); Lik (Paithe); Maram (Tangkhul); Peikashasthao (Kabui, Rongmei).

Distribution: Arunachal Pradesh, Assam, Manipur, Meghalaya, Mizoram, Nagaland, Sikkim, West Bengal. [China, Myanmar].

Chimonocalamus longiusculus Hsueh \& T.P.Yi, Acta Bot. Yunnan. 1(2): 80. 1979.

Type: n.v.

Distribution: Arunachal Pradesh. [Yunnan].

Chimonocalamus lushaiensis Ohrnb., Bamb. World Introd. Ed. 4: 314. 1997. Sinarundinaria longispiculata C.S.Chao \& Renvoize, Kew Bull. 43: 411, 1988.

Type: India: Assam, Sangao, Lushai Hills, 1300 m, March 1953, Thakur Rup Chand 6889 (K).

Distribution: Mizoram. Endemic.

Chimonocalamus nagalandianus (H.B.Naithani) P.Kumari comb. nov. Sinarundinaria nagalandiana H.B.Naithani, Indian Forester 120(12): 1120. 1994.

Type: India: Nagaland, Niriyo Peak, alt. 3840 ft., Wokha, November 1986, H.B.Naithani 1477 (Holo DD).

Vern.: Nkyip (Naga).

Distribution: Nagaland. Endemic.

DENDROCALAMUS Nees, Linnaea 9: 476. 1835.

Type: Dendrocalamus strictus (Roxb.) Nees (Bambusa striata Roxb.)

Dendrocalamus brandisii (Munro) Kurz, Forest Fl. Burma 2: 560. 1877. Bambusa brandisii Munro, Trans. Linn. Soc. London 26(1): 109-110. 1868.

Type: "Hab. in Ind. or. Tenasserim, Martaban, Pegu, usque ad 4000 ped. S.M., praecipue in saxis Calcareis, Brandis no. 2. (HBG).

Vern.: Bulka (Beng.); Wanan (Manipur).

Distribution: Andaman \& Nicobar Islands, Kerala (TBGRI), Manipur. [China, Myanmar].

Dendrocalamus calostachyus (Kurz) Kurz, Forest Fl. Burma 2: 562. 1877. Bambusa calostachya Kurz, J. Asiat. Soc. Bengal Pt. 2. Nat. Hist 42: 250. 1873.

Type: Burma: Ava, Bhamo, Kakhyen Hills, alt. 3500 ft., Kurz s.n.

Distribution: Meghalaya, Nagaland. [China, Myanmar]. 
Dendrocalamus giganteus Munro, Trans. Linn. Soc. London 26(1): 150. 1868.

Lectotype: 'Cult. Calcutta', Hb. Munro (K). LT designated by Stapleton in Edinburg J. Bot. 51 (1): 23. 1994.

Vern.: Worra (Ass.); Maroobob (Manipuri); U-ktang (Khasi).

Distribution: Arunachal Pradesh, Assam, Karnataka, Maharashtra, Meghalaya, Mizoram, Nagaland, Uttarakhand, West Bengal. [China, Myanmar, Indonesia, Nepal, Sri Lanka].

Dendrocalamus hamiltonii Nees \& Arn. ex Munro, Trans. Linn. Soc. London 26(1): 151. 1868.

Lectotype: India: Assam, Goalpara, July 17, 1808, BuchananHamilton 882 (E). LT designated by Stapleton in Edinburg J. Bot. 51(1): 23. 1994.

Vern.: Ka-Sejlei (Khasi); Wanok (Garo); Peidiau (Kabui, Rongmai); Pecha (Jiribam); Wanap, Unap (Manipuri); Kongha, Ramkaha (Tangkhul); Gova, Gonam (Kuki); Phulrua (Mizo); Ravaw, Arua, Ravawng (Mara); Rawpui (Paite); Uaktormah (Bru/ Riang).

Distribution: Arunachal Pradesh, Assam, Bihar, Himachal Pradesh, Manipur, Meghalaya, Mizoram, Nagaland, Sikkim, Tripura, Uttarakhand. [Bangladesh, Bhutan, China, Myanmar, Nepal].

Dendrocalamus hookeri Munro, Trans. Linn. Soc. London 26(1): 151. 1868.

Lectotype: India: Bengal orient. Pundua, June 1l, 1850, J.D.Hooker \& Thomson 411 (K). LT designated by Stapleton in Edinburg J. Bot. 5 l (1): 25. 1994.

Vern.: Seiat, Sejsai, Sijong, Ukotang (Ass.); Siejong, Ussey (Khasi); Denga (Garo); Patu (Lepcha); Ooer (Manipuri); Tili bans(Nepal); Rawpui, Rawlak, Rawkhauh (Mizo).

Distribution: Arunachal Pradesh, Assam, Manipur, Meghalaya, Mizoram, Nagaland, Sikkim, West Bengal. [Myanmar, Nepal].

Dendrocalamus hookeri Munro var. parishii (Munro) Blatt., Indian Forester 55( 11): 594. 1929. Dendrocalamus parishii Munro, Trans. Linn. Soc. London 26(1): 149. 1868.

Type: India: Punjab Himalaya, Parish s.n. (K).

Distribution: Arunachal Pradesh, Assam, Himachal Pradesh, Manipur, Meghalaya, Mizoram, Nagaland, Punjab, Sikkim, West Bengal. [China, Myanmar, Nepal, Pakistan].

Dendrocalamus latiflorus Munro, Trans. Linn. Soc. London 26(l): 152.1868

Syntypes: Hab. In China. Hongkong: Hance, 1050; Haviland 740; Formosa, R.Oldham 649 (K).

Vern.: Hava, Kaha (Tangkhul); Gomi (Kuki, Paite); Maribob (Manipuri).

Distribution: Manipur, Mizoram, Nagaland. [Yunnan, Taiwan, Myanmar, Vietnam].
Dendrocalamus longispathus Kurz, Forest Fl. Burma 2: 561. 1877.

Lectotype: Myanmar, 1871, Kurz s.n. (K). Lectotype selected by M.Kumar in KFRI Research Report no. 330: 27. 2009.

Vern.: Kako (Khasi); Rupai (Tripura); Wui, Uil (Manipuri); Gothi (Paite); Dujangpai (Kabui); Rawnal (Mizo); Rabnia (Mara); Pharbuabas (Chakma); Unamlih (Bru/ Riang).

Distribution: Assam, Jharkhand, Meghalaya, Mizoram, Orissa, Tripura, Uttarakhand. [Bangladesh, China, Myanmar, Thailand].

Dendrocalamus manipureanus H.B.Naithani, Indian Forester 136(2): 262-265. 2010.

Type: India: Manipur, Moreh, Sinaiveng, 10.10.2008, H.B.Naithani 5007 (Holo DD).

Vern.: Nango (Kuki); Ooe, Oei, Uii (Manipuri); Ruv (Machi); Rawchhe, Rawchhe-changdam (Mizo).

Distribution: Manipur.

Dendrocalamus membranaceus Munro, Trans. Linn. Soc. London 26(1): 149. 1868. Bambusa membranacea (Munro) Stapleton \& N.H. Xia, Kew Bull. 52(1): 238. 1997.

Lectotype: Burma: Martaban, Trogla, March 12, 1827, Numer. List [Wallich] no 5029 (K). LT designated by Stapleton \& N.H. Xia in Kew Bull. 52: 238. 1097.

Distribution: Kerala, West Bengal. [China, Myanmar].

Dendrocalamus sahnii H.B.Naithani \& Bahadur, Indian Forester 108(3): 212. f. 1. 1982.

Type: India: Arunachal Pradesh, Subansiri District, Zoram, alt. 6000 ft., April 28, 1977, H.B.Naithani ser II no. 902 (DD).

Distribution: Arunachal Pradesh. Endemic.

Dendrocalamus sikkimensis Gamble ex Oliver, Hooker's Icon. Pl. 18: t. 1770. 1888.

Lectotype: India: Sikkim, July 20, 1885, Pantling s.n. (K). LT designated by Stapleton in Edinburg J. Bot. 51 (1) : 25. 1994. Vern.: Wadah (Garo); Pugriang (Lepcha); Rawami, Sangau (Mizo); Anong, Amoi, Ano (Mara); Paalm (Kabui, Rongmei); Bhalu bans (Nepali).

Distribution: Assam, Arunachal Pradesh, Meghalaya, Nagaland, Sikkim, West Bengal. [Bhutan, China, Nepal].

Dendrocalamus somdevae H.B.Naithani, Indian Forester 119(6): 504. 1993.

Type: India: Dehra Dun, Hardwar road between Jogiwala and Majri (Mokhampur), 11.3.1991, Som Deva 10985 (Holo DD); H.B.Naithani 1694 (Para DD); Circuit house Almora (Kumaun), 9.8.1991, H.B.Naithani 1722 (Para DD).

Distribution: Uttarakhand. Endemic.

Dendrocalamus strictus (Roxb.) Nees, Linnaea 9: 476-477. 1834. Bambos stricta Roxb., Pl. Coromandel 1: 58. t. 80. 1795.

Type: Roxb., Pl. Coromondel 1: 58, t. 80. 1798. 
Vern.: Karali (Beng.); Nakur bans, Kiri bidiru (Gujrat); Male bamboo, Narvel (Maharashtra); Salia (Orrisa); Kalmungil (Tamil); Sadanapa Veduru (Telugu); Lathi bans (Tripura); Kallumua (Kerala).

Distribution: Andhra Pradesh, Arunachal Pradesh, Assam, Bihar, Chhatisgarh, Dadra \& Nagar Haveli, Gujarat, Himachal Pradesh, Jharkhand, Karnataka, Kerala, Madhya Pradesh, Maharashtra, Meghalaya, Mizoram, Orissa, Rajasthan, Tamil Nadu, Uttar Pradesh. [Bangladesh, China, Pakistan, Nepal].

Dendrocalamus strictus (Roxb.) Nees var. sericeus (Munro) Gamble, Man. Ind. Timb. ed. 2. 751. 1902. Dendrocalamus sericeus Munro, Trans. Linn. Soc. London 26(1): 148. 1868

Syntypes: India: Behar [Bihar], Parasnath, alt. $4000 \mathrm{ft}$, September, 1858, Thomson s.n. (K). India: Behar [Bihar], Parasnath, February 1848, Hooker s.n. (K).

Distribution: Bihar. Endemic.

DINOCHLOA Buse, Gram. (Buse) 47. 1854.

Type: Dinochloa tjankorreh Buse, nom. illeg. (Bambusa scandens Blume, Dinochloa scandens (Blume) Kuntze).

Dinochloa andamanica Kurz, J. Asiat. Soc. Bengal Pt. 2. Nat. Hist. 42: 253. 1873

Type: India: "Andamans (etiam in insula Nicobarieis)"

Vern.: Baradahbarat, Bulu akar (Andaman).

Distribution: Andaman \& Nicobar Islands. [Myanmar, Thailand].

Dinochloa maclellandii (Munro) Kurz, J. Asiat. Soc. Bengal Pt. 2. Nat. Hist. 42: 253. 1873. Bambusa macclellandii Munro, Trans. Linn. Soc. London 26(1): 114. 1868.

Type: "Hab in Ind. or Pegu, Rangon, Phoungee Valley, Mc'Clelland".

Distribution: Arunachal Pradesh, Assam, Ultarakhand, West Bengal. [Bangladesh, Myanmar, Thailand, Vietnam].

Dinochloa nicobariana R.B.Majumdar in Karthik. et al., Fl. Ind. Enumerat.- Monocot. 277. 1989.

Type: India: Nicobar Islands, North Nicobar, Katchal Island, Coastal Forest, P.Chakravarty 1129 (CAL).

Distribution: Nicobar Islands. Endemic.

DREPANOSTACHYUM Keng f., J. Bamboo Res. 2(1): 15. 1983; Stapleton, Fl. Bhutan 3(2): 505. 2000.

Type: Drepanostachyum falcatum (Nees) Keng f. (Arundinaria falcata Nees).

Drepanostachyum falcatum (Nees) Keng f., J. Bamboo Res. 2(1): 16. 1983. Arundinaria falcata Nees, Linnaea 9: 478. 1835. Sinarundinaria falcata (Nees) C.S.Chao \& Renvoize, Kew Bull. 44(2): 357. 1989.
Lectotype: Northwest India, Royle s.n. (K). LT designated by C.S.Chao \& Renvoize, Kew Bull. 44(2): 358. 1989.

Vern.: Ringal (Garhwali); Leik (Mizo); Syuli (Mara); Simtong (Kuki); Machum (Tangkhul).

Distribution: Himachal Pradesh, Mizoram, Uttarakhand. [Combodia, Laos, Nepal, Vietnam].

Drepanostachyum intermedium (Munro) Keng f., J. Bamboo Res. 2(1): 18. 1983. Arundinaria intermedia Munro, Trans. Linn. Soc. London 26(1): 28. 1868. Sinarundinaria intermedia (Munro) C.S.Chao \& Renvoize, Kew Bull. 44(2): 357. 1989.

Type: India: Sikkim, alt. 7000-8000 ft., 1848, J.D.Hooker s.n. (K).

Vern.: Purmiok, Purmiak, Permyok, Parmiok, Prongnok, Purmia (Lepcha); Tite, Titi, Titay, Ningalo, Nigalai (Nepali); Lik (Lushai).

Distribution: Arunachal Pradesh, Meghalaya, Mizoram, Sikkim, West Bengal. [Bhutan, Nepal].

Drepanostachyum khasianum (Munro) Keng f., J. Bamboo Res. 2(1): 18. 1983. Arundinaria khasiana Munro, Trans. Linn. Soc. London 26(1): 28. 1868.

Type: India: Khasia, Shillong, 5800 ft., Griffith 1058 (K).

Vern.: Nam-long (Khasi).

Distribution: Assam, Meghalaya, Sikkim. [Bhutan, Nepal].

Drepanostachyum kurzii (Gamble) Pandey ex D.N. Tewari, Monogr. Bamboo 90. 1993. Arundinaria kurzii Gamble, Ann. Roy. Bot. Gard. Calcutta 7: 25. t. 25. 1896. Sinarundinaria kurzii (Gamble) M. Kumar, Bamboos of India Compendium 279. 1998.

Type: Burma: Southern coast, 1878, Kurzs.n. (K).

Distribution: Manipur, Meghalaya, Nagaland. [Myanmar].

Drepanostachyum polystachyum (Kurz ex Gamble) R.B.Majumdar in Karthik. et al., Fl. Ind. Enumerat. Monocot. 277. 1989. Arundinaria polystachya Kurz ex Gamble, Ann. Roy. Bot. Gard. Calcutta 7: 7. t. 5. 1896. Sinarundinaria polystachya (Kurz ex Gamble) C.S.Chao \& Renvoize, Kew Bull. 44(2): 359. 1989.

Lectotype: India: Sikkim, alt. 4000-5000 ft., 1868, Kurz \& Andrson s.n. (K). LT designated by C.S.Chao \& Renvoize, Kew Bull. 44(2): 359. 1989.

Distribution: Meghalaya, Sikkim. Endemic.

Drepanostachyum suberectum (Munro) R.B.Majumdar, Bull. Bot. Surv. India 25(1-4): 236. 1983. Arundinaria suberecta Munro, Trans. Linn. Soc. London 26(1): 32. 1868. Sinarundinaria suberecta (Munro) M. Kumar, Bamboos of India Compendium 286. 1998. 
Lectotype: India: Meghalaya, Mamlo, October 27, 1835, Griffith 558 (K). LT designated by Stapleton, Edinburgh J. Bot. 51: 308. 1994.

Vern.: Ukadai Namlang, Lamb-nag (Jaintia); Nam-long (Khasi).

Distribution: Arunachal Pradesh, Meghalaya, Sikkim. Endemic.

GIGANTOCHLOA Kurz ex Munro, Trans. Linn. Soc. London 26: 123. 1868.

Lectotype: Gigantochloa atter Kurz ex Munro (Bambusa thouarsii var. atter Hassk.). LT designated by Holttum in Taxon 5: 28-30. 1956.

Gigantochloa albociliata (Munro) Kurz, Forest Fl. Burma 2: 555. 1877. Oxytenanthera albociliata Munro, Trans. Linn. Soc. London 26 (1): 129. 1868.

Syntypes: "Hab. in India or." [Burma]: Pegu, Brandis 19 ("Wapyoogle" incolis) (K); India: Moulmein, Falconer 27. (K). Distribution: Arunachal Pradesh, Assam, Meghalaya, Tripura, West Bengal. [Bangladesh, China, Laos, Myanmar, Thailand, Vietnam]. Naturalized.

Gigantochloa andamanica (Kurz) Kurz, Prelim. Rep. For. Veg. Pegu, 1875. Bambusa andamanica Kurz, J. Asiat. Soc. Bengal n.s. 39 (2): 88. 1870.

Type: Andaman Islands: south Andaman, Kurzs.n.

Vern.: Chatai bamboo (Andaman), Ootal (Andamani); Pangnu, Pui (Kabui, Rongmei).

Distribution: Andaman Islands. [Bangladesh, Jawa, Laos, Myanmar, Sumatra, Thailand, Vietnam].

Gigantochloa apus (Schult. \& Schult. f.) Kurz, Natuurk. Tijdschr. Ned.-Indie 27: 226. 1864. Bambusa apus Schult. \& Schult. f., Syst. Veg. 7(2): 1353. 1830.

Type: Indonesia: Java, Salaki, Blume s.n. (Holo, M; Iso, L). Widjaja, Reinwardtia 10(3): 349. 1987.

Vern.: Tekserah, Washut (Garo).

Distribution: Meghalaya. [Bangladesh, Borneo, Jawa, Laos, Malaya, Myanmar, Thailand].

Gigantochloa auriculata (Kurz) Kurz, Forest Fl. Burma 2: 557. 1877. Bambusa auriculata Kurz, J. Asiat. Soc. Bengal Pt. 2. Nat. Hist 39: 86. 1870.

Type: Burma: Southern Pegu, Kurz 20 (CAL).

Distribution: Assam, West Bengal. [Bangladesh, Myanmar].

Gigantochloa bastareana H.B. Naithani \& R.C. Pal, in Indian Forester 136(9): 1276-1277. 2010.

Type: India: Chhattisgarh, Bastar district, Kanger Valley National Park, Dandak Gupha, 02.11.2004, H.B.Naithani 4298 (Holo DD).

Vern.: Peeta (Kanger valley).

Distribution: Chhattisgarh.
HIMALAYACALAMUS Keng f., J. Bamboo Res. 2(1): 23. 1983.

Type: Himalayacalamus falconeri (Hook.f. ex Munro) Keng f. (Thamnocalamus falconeri Hook.f. ex Munro)

Himalayacalamus falconeri (Hook.f. ex Munro) Keng f., J. Bamboo Res. 2(1): 24. 1983. Thamnocalamus falconeri Hook.f. ex Munro, Trans. Linn. Soc. London 26(1): 34. 1868.

Type: Nepal: Bagmati Zone, Kathmandu, Sheopore [Shivapuri], alt. 8000 ft., 1821, Numer. List [Wallich] no. 5040 (K).

Vern.: Deo-ringal, Dev-ringal, Ningal (Garhwal); Singhane, Maling (Darjeeling); Pao-muarg, Purnoon (Sikkim).

Distribution: Sikkim, Uttar Pradesh, West Bengal. [Bhutan, China, Nepal].

Himalayacalamus hookerianus (Munro) Stapleton, Bamboo Soc. Newsl. 17: 21. 1993. Arundinaria hookeriana Munro, Trans. Linn. Soc. London 26(1): 29. 1868. Sinarundinaria hookeriana (Munro) C.S.Chao \& Renvoize, Kew Bull. 44(2): 358. 1989. Drepanostachyum hookerianum (Munro) Keng f., J. Bamboo Res. 2(1): 17. 1983. Chimonobambusa hookeriana (Munro) Nakai, J. Arnold Arbor. 6(3): 151. 1925.

Lectotype: India: Sikkim [Neongong, 6800 ft.], "Praong, 4000-6000 ft.", December 1848, J.D.Hooker s.n. (K). LT designated by Stapleton in Edinburgh J. Bot. 51: 318. 1994.

Vern.: Praong, Prong, Praing, Preng, Pareng (Lepcha); Singhane, Singhani, Suighani (Nepal).

Distribution: Arunachal Pradesh, Meghalaya, Sikkim, West Bengal. [Bhutan].

KURUNA Attigala, Kathr. \& L.G.Clark, Phytotaxa 174(4): 182. 2014.

Type: Arundinaria debilis Thwaites

Kuruna densifolia (Munro) Attigala, Kathr. \& L.G.Clark, Phytotaxa 174(4): 200. 2014. Arundinaria densifolia Munro, Trans. Linn. Soc. London 26(1): 32. 1868. Sinarundinaria densifolia (Munro) C.S.Chao \& Renvoize, Kew Bull. 44(2): 354. 1989. Yushania densifolia (Munro) R.B.Majumdar in Karthik. et al., Fl. Ind. Enumerat.- Monocot. 282. 1989. Chimonobambusa densifolia (Munro) Nakai, J. Arnold Arbor. 6: 151. 1925.

Lectotype: Sri Lanka: Watson 25 (K). LT designated by Soderstrom \& Ellis in Smithsonian Contr. Bot. 72: 12. 1988.

Distribution: Kerala. [Sri Lanka].

Kuruna floribunda (Thwaites) Attigala, Kathr. \& L.G.Clark, Phytotaxa 174(4): 200. 2014. Indocalamus floribundus (Thwaites) Nakai, J. Arnold Arbor. 6: 148. 1925. Arundinaria floribunda Thwaites, Enum. Pl. Zeyl. (Thwaites) 375. 1864. Sinarundinaria floribunda (Thwaites) C.S.Chao \& 
Renvoize, Kew Bull. 44(2): 356. 1989. Yushania floribunda (Thwaites) Demoly, Bambou 48: 12. 2006.

Type: Sri Lanka: Maturatte District, alt. 5000 ft., 1863, Thwaites Ceylon Plant 2624 (PDA).

Distribution: Kerala, Tamil Nadu. [Sri Lanka].

Kuruna walkeriana (Munro) Attigala, Kathr. \& L.G.Clark, Phytotaxa 174(4): 200. 2014. Indocalamus walkerianus (Munro) Nakai, J. Arnold Arbor. 6: 148. 1925. Arundinaria walkeriana Munro, Trans. Linn. Soc. London 26(1): 21. 1868. Sinarundinaria walkeriana (Munro) C.S.Chao \& Renvoize, Kew Bull. 44(2): 354. 1989. Yushania walkeriana (Munro) R.B.Majumdar in Karthik. et al., Fl. Ind. Enumerat.Monocot. 283. 1989.

Lectotype: Sri Lanka: Mrs. Walker 96 (K). LT designated by Soderstrom \& Ellis in Smithsonian Contr. Bot. 72: 27. 1988.

Distribution: Kerala, Tamil Nadu. [Sri Lanka].

Kuruna wightiana (Nees) Attigala, Kathr. \& L.G.Clark, Syst. Bot. 4l(1): 191. 2016. Indocalamus wightianus (Nees) Nakai, J. Arnold Arbor. 6: 149. 1925. Arundinaria wightiana Nees, Linnaea 9: 482. 1834. Sinarundinaria wightiana (Nees) C.S.Chao \& Renvoize, Kew Bull. 44(2): 356. 1989. Yushania wightiana (Nees) R.B.Majumdar in Karthik. et al., Fl. Ind. Enumerat.Monocot. 283. 1989.

Type: India: Nilgiri District: Wight 1797 (Holo CAL, Iso K).

Vern.: Chewari (Kerala, Tamil Nadu).

Distribution: Kerala, Tamil Nadu. [Sri Lanka].

Kuruna wightianus (Nees) Attigala, Kathr. \& L.G.Clark var. hispida P.Kumari comb. nov. Indocalamus wightianus (Nees) Nakai var. hispida (Steud.) Nakai, J. Arnold Arbor. 6: 149. 1925. Arundinaria wightiana Nees var. hispida (Steud.) Gamble, Ann. Roy. Bot. Gard. Calcutta 7: 5. 1896. Yushania wightiana (Nees) R.B.Majumdar var. hispida (Steud.) R.B.Majumdar in Karthik. et al., Fl. Ind. Enumerat.Monocot. 283. 1989. Arundinaria hispida Steud., Syn. Pl. Glumac. I: 335. 1854.

Type: India: "In sylvis prope Sispara Nilagiri", Hohenacker 1282 (K).

Distribution: Kerala, Tamil Nadu. [Sri Lanka].

MELOCALAMUS Benth. in Benth. \& J.D. Hooker, Gen. Pl 3, 2: 1095, 1212. 1883.

Type: Melocalamus compactiflorus (Kurz) Benth.

Melocalamus compactiflorus (Kurz) Benth., Gen. Pl. (Benth. \& Hook.f.) 3: 1212. 1883.

Pseudostachyum compactiflorum Kurz, J. Asiat. Soc. Bengal Pt. 2. Nat. Hist. 42: 252. 1873.

Dinochloa compactiflora (Kurz) McClure, Bull. Misc. Inform. Kew 1936: 253. 1936. Melocalamus mastersii (Munro) R.B.Majumdar in Karthik. et al., Fl. Ind. Enumerat.-
Monocot. 278. 1989. Bambusa mastersii Munro, Trans. Linn. Soc. London 26(1): 113. 1868. Type: Martaban, Burma, S.Kurz 3183 (CAL).

Vern.: Lata-bans (Ass.); Dural, Doral (Beng.); Sairil (Mizo); Sairi (Mara); Uadu (Bru/ Riang); Chhairil (Lai).

Distribution: Assam, Manipur, Meghalaya, Mizoram, Tripura. [Bangladesh, China, Laos, Myanmar, Thailand, Vietnam].

Melocalamus gracilis R.B.Majumdar in Karthik. et al., Fl. Ind. Enumerat.- Monocot. 278. 1989. Dinochloa gracilis (R.B.Majumdar) Bennet \& Jain ex D.N. Tewari, Monogr. Bamboo 82. 1992.

Type: India: Barail Range, near Kailana, 9 km from Gumri rest house on Shillong-Cachar Road near P.W.D. Shed, R.B.Majumdar 1138 (CAL).

Distribution: Assam. Endemic.

Melocalamus indicus R.B.Majumdar, Bull. Bot. Surv. India 25(1-4): 236. 1983. Dinochloa indica (R.B.Majumdar) Bennet, Van Vigyan 27(2): 121. 1989.

Type: India: Cachar, Bhuban Hills, R.B.Majumdar 73083 (CAL).

Vern.: Dural (Beng.); Gilching (Kuki), Uma (Manipuri); Charih (Rongmei).

Distribution: Assam, Manipur. Endemic.

MELOCANNA Trin. in K.P.J. Sprengel, Neue Entdeck. Pflanzenk. 2: 43. 1821.

Type: Melocanna bambusoides Trin., nom, illeg. (Bambusa baccifera Roxb.; Melocanna baccifera (Roxb.) Kurz)

Melocanna baccifera (Roxb.) Kurz, Prelim. Rep. Forest Pegu 94. 1875. Bambusa baccifera Roxb., Pl. Coromandel 3: 37, t. 243. 1819. Melocanna humilis Kurz, Forest Fl. Burma 2: 569. 1877, non Roep. ex Trin. 1822. Melocanna arundina C.E.Parkinson, Indian Forester 61: 326. 1935.

Lectotype: Roxburgh Icones 1401 (K). LT designated by Stapleton in Edinburgh J. Bot. 5l: 27. 1994.

Vern.: Muli, U-silli (Khasi); Wah-thri, Wa-sith, Watray, Warai (Garo); Moubi-wa (Manipuri); Mautak, Maomitvel (Mizo); Ramaw (Mara); Egochiabas (Chakma); Uarthoi (Bru/ Riang); Mou (Paithe).

Distribution: Assam, Karnataka, Maharashtra, Manipur, Meghalaya, Mizoram, Sikkim, Tripura, West Bengal. [Bangladesh, China, Myanmar, Nepal].

Melocanna clarkei (Gamble ex Brandis) P.Kumari \& P.Singh, Nelumbo 5l: 234. 2009. Arundinaria clarkei Gamble ex Brandis in Ind. Trees 666. 1906. Schizostachyum mannii R.B.Majumdar in Karthik. et al., Fl. Ind. Enumerat.Monocot. 281. 1989. Bambusa khasiana sensu Gamble, Ann. Roy. Bot. Gard. Calcutta 7: 39. t. 37. 1896 non Munro, 1868. 
Type: Cherra end, 2000 ft., Oct. 1867, C.B.Clarke 5563! (Holo CAL).

Vern.: Rawte, Chatle (Mizo); Uma (Manipuri); Chi (Kabui); Gehour (Jiribam); Inchi (Rongmei).

Distribution: Assam, Manipur, Meghalaya, Mizoram, Nagaland. Endemic.

MUNROCHLOA M.Kumar \& Remesh, J. Bot. Res. Inst. Texas (1): 374. 2008.

Type: Bambusa ritcheyi Munro

Munrochloa ritchiei (Munro) M.Kumar \& Remesh, J. Bot. Res. Inst. Texas (1): 374. 2008. Bambusa ritcheyi Munro, Trans. Linn. Soc. London 26(1): 113.1868. Pseudoxytenanthera ritcheyi (Munro) H.B.Naithani, J. Bombay Nat. Hist. Soc. 87(3): 440. 1990.

Type: India: Bombay, Kala Nuddi, Ritchie 820 (W).

Vern.: Choomaree, Choua, Chiwa, Chiwan, Chawa (Kannad); Huda, Udhe, Manga, Tandali (Marathi).

Distribution: Karnataka, Kerala, Maharashtra, Tamil Nadu. Endemic.

NEOHOUZEAUA A. Camus, Bull. Mus. Nat. Hist. Paris 28: 100. 1922.

Type: Neohouzeaua mekongensis A. Camus

Neohouzeaua dullooa (Gamble) A.Camus, Bull. Mus. Hist. Nat. (Paris) 28: 100. 1922. Teinostachyum dullooa Gamble, Ann. Roy. Bot. Gard. Calcutta 7: 101. 1896. Schizostachyum dullooa (Gamble) R.B.Majumdar in Karthik. et al., Fl. Ind. Enumerat.- Monocot. 281. 1989.

Type: Burma: Katha District, Hawyaw Monastery Garden, February 1892, Oliver s.n. (K).

Vern.: Dullooa (Ass.); Puksalu (Lepcha); Dongla (Khasi); Wadroo (Garo); Rawthlaw (Mizo); Uatlau (Bru); Dhulubans (Chakma); Gu, Goo (Kabui, Rongmei); Nat, Tolu, Phisautong (Manipuri); Gokhoo (Paite); Sedlar (Maring); Shia (Tangkhul).

Distribution: Assam, Manipur, Meghalaya, Mizoram, Nagaland, Sikkim, Uttarakhand, West Bengal. [Bangladesh, Bhutan, Myanmar].

Neohouzeaua helferi (Munro) Gamble, Bull. Misc. Inform. Kew 1923: 91. 1923. Bambusa helferi Munro, Trans. Linn. Soc. London 26(1): 114. 1868. Pseudostachyum helferi (Munro) Kurz, J. Asiat. Soc. Bengal Pt. 2, Nat. Hist. 42: 253. 1872. Teinostachyum helferi (Munro) Gamble, Ann. Roy. Bot. Gard. Calcutta 7: 102. t. 90. 1896. Schizostachyum helferi (Munro) R.B.Majumdar in Karthik. et al., Fl. Ind. Enumerat.Monocot. 281. 1989.

Type: Burma: Tenasserim, Helfer 411 (K).

Vern.: Silloh (Khasi); Wali, Tumoh (Garo).

Distribution: Arunachal Pradesh, Assam, Meghalaya. [Bangladesh, Myanmar].
NEOMICROCALAMUS P.C. Keng, J. Bamboo Res. 2(2): 146. 1983.

Type: Neomicrocalamus prainii (Gamble) Keng f. (Microcalamus prainii Gamble)

Neomicrocalamus andropogonifolius (Griff.) Stapleton, Edinburgh J. Bot. 51(3): 325. 1994. Bambusa andropogonifolia Griff., Itin. Pl. Khasyah Mts. 124. 1848.

Type: Bhutan: Tashigang District, Diri Chhu, $\left[27^{\circ} 10^{\circ} \mathrm{N} .91^{\circ}\right.$ $26^{\circ} \mathrm{E}$ ], alt. $3600 \mathrm{ft}$. Griffith Itin 417 (K).

Distribution: Nagaland. [Bhutan].

Neomicrocalamus mannii (Gamble) R.B.Majumdar in Karthik. et al., Fl. Ind. Enumerat.- Monocot. 279. 1989. Cephalostachyum mannii (Gamble) Stapleton \& D.Z.Li, Kew Bull. 52: 700. 1997. Arundinaria mannii Gamble, Ann. Roy. Bot. Gard. Calcutta 7: 26. 1896. Racemobambos mannii (Gamble) M.Kumar, Bamboos of India Compendium 230. 1998.

Type: India: Meghalaya, Amkasur, Jaintia Hills, 3000 ft., April 24, 1889, G.Mann 21845 (K).

Vern.: Beneng, Ka-beneng (Jaintia \& Khasi); Siaman (Mizo). Distribution: Meghalaya. [China].

Neomicrocalamus prainii (Gamble) Keng f., J. Bamboo Res. 2(2): 10. 1983. Microcalamus prainii Gamble, J. Asiat. Soc. Bengal Pt. 2, Nat. Hist. 59(2): 207. t. 7. 1891. Arundinaria prainii (Gamble) Gamble, Ann. Roy. Bot. Gard. Calcutta 7: 2l. t. 19. 1896. Racemobambos prainii (Gamble) Keng f. \& T.H.Wen, J. Bamboo Res. 5(2): 13. 1986.

Type: India: Assam, Naga Hills, 8300 ft., April 23, 1886, Prain s.n. (K).

Vern.: U-speit (Khasi); Tenwa (Manipuri); Sinar (Tangkhul).

Distribution: Assam, Meghalaya, Nagaland. [China].

OCHLANDRA Thwaites, Enum. Pl. Zeyl. (Thwaites). 376. 1864.

Type: Ochlandra stridula Thwaites

Ochlandra beddomei Gamble, Ann. Roy. Bot. Gard. Calcutta 7: 124. t. 110. 1896.

Type: India: Wet slopes of Nilgiris-Wynaad, 3000-4500ft., Beddome s.n. (K). LT designated by M.Kumar in KFRI Research Report no. 399: 84. 2011.

Distribution: Kerala. Endemic.

Ochlandra ebracteata Raizada \& Chatterjee, Indian Forester 89(5): 362. 1963.

Type: India: Kerala, Paruthipally range, Kottur reserve, Trivandrum division, June 28, 1961, Managing Agents, Punalur Paper Mills s.n, (Holo, DD).

Distribution: Kerala. Endemic.

Ochlandra keralensis M.Kumar, Remesh \& Sequiera, J. Econ. Taxon. Bot. 25(1): 49-5l.f. 1 (A-N). 2001. 
Type: India: Kerala, Pathanamthitta District, Pachakkanam, 3200 ft., December 9, 1998, Remesh \& Stephen 20730 (Holo, KFRI).

Distribution: Kerala. Endemic.

Ochlandra scriptoria (Dennst.) C.E.C.Fiseh., Fl. Madras 3(10): 1863. 1934. Bambusa scriptoria Dennst., Schltissel Hort. Malab. 31. 1818. Ochlandra rheedii (Kunth) Benth. \& Hook.f. ex Gamble, Ann. Roy. Bot. Gard. Calcutta 7: 121. t. 107. 1896. Beesha rheedii Kunth, Enum. Pl. 1: 434, 1833.

Type: Rheede, Hortus. Malab. 3. t. 60 as "Beesha". Vern.: Ammei, Bheesha, Kolanji, Ottal (Malyali). Distribution: Karnataka, Kerala, Tamil Nadu. Endemic.

Ochlandra setigera Gamble, Ann. Roy. Bot. Gard. Calcutta 7: 128. t. 115. 1896.

Type: India: western slopes of Nilgiri Hills, Gudalur, alt. 3000 ft., H.Trimen s.n.; j.S.Gamble 25503 (Holo K).

Distribution: Kerala, Tamil Nadu. Endemic.

Ochlandra spirostylis M.Kumar, K.K.Seethal. \& Sequiera, Rheedea 9(1): 31-33. 1999.

Type: India: Kerala, Idukki District, Adimali, Chattuparakudy, 3000 ft., May 30, 1998, Stephen Sequiera 00884 (Holo, KFRI).

Distribution: Kerala. Endemic.

Ochlandra talbotii Brandis, Indian Trees 684. 1906.

Lectotype: India: North Canara, Gursoppa falls, W.A.Talbot 3628 (Lecto, BLAT).

Distribution: Karnataka, Goa. Endemic.

Ochlandra travancorica (Bedel.) Benth. ex Gamble, Ann. Roy. Bot. Gard. Calcutta 7: 125. t. 111. 1896. Beesha travancorica Bedd., Forester's Man. Bot. 234. 1873. Ochlandra sivagiriana (Gamble) E.G.Camus, Bambusees (Camus) 181.1. 99. f. c. 1913. Ochlandra rheedii Benth. ex Gamble var. sivagiriana Gamble, Ann. Roy. Bot. Gard. Calcutta 7: 122. t. 108. 1896. Ochlandra soderstromiana M.Kumar \& Sequiera, Rheedea 9(1): 33. f. 2. 1999. Ochlandra kadambarnii M.Kumar, Unnikrishnana $\&$ Remesh, Bamboos of Penninsular India, KFRI Research report no. 399: 92. 2011. Ochlandra travancorica (Bedd.) Benth. ex Gamble var. hirsuta Gamble, Ann. Roy. Bot. Gard. Calcutta 7: 126. t. 112. 1896.

Type: India: Mountains of Tinnevelly and Travancore, alt. 3000-5000 ft., Madras, Beddome s.n. (K).

Vern.: Etta, Kar-eetta, Vei (Malyali); Eeral, Eera-kalli, Iral, Irul, Ita-kalli, Nanal, Odai (Tamil).

Distribution: Karnataka, Kerala, Tamil Nadu. Endemic.

Ochlandra wightii (Munro) C.E.C.Fisch., Fl. Madras 3(10): 1864. 1934. Bambusa wightii Munro, Trans. Linn. Soc. London 26(1): 11l. 1868. Ochlandra brandisii Gamble, Ann. Roy. Bot. Gard. Calcutta 7: 126. t. 1 13. 1896.
Type: India: Courtallum, alt. 3000 ft., 1835, Brandis s.n. (Neotype, K). Designated by M.Kumar in KFRI Research Report no. 399: 121. 2011.

Vern.: Ira-calli (Tamil).

Distribution: Kerala, Tamil Nadu. Endemic.

PHYLLOSTACHYS Siebold \& Zucc., Abh. Math.-Phys. Cl. Konigl. Bayer. Akad. Wiss. 3: 745. 1843, nom. cons.

Type: Phyllostachys bambusoides Siebold \& Zucc.

Phyllostachys bambusoides Siebold \& Zucc., Abh. Math. Phys. Cl. Konigl. Bayer. Akad. Wiss. 3(3): 746. pl. 5. f. 3. 1843.

Type: Japan; P.F. von Siebold s.n. (L).

Vern.: Makade (Japanese).

Distribution: Assam, Himachal Pradesh, Sikkim. [China]. Cultivated and possibly naturalized, native to China and Japan.

Phyllostachys mannii Gamble, Ann. Roy. Bot. Gard. Calcutta 7: 28. t. 28. 1896. Phyllostachys assamica Gamble ex Brandis, Indian Trees 667. 1906. Phyllostachys bambusoides sensu Gamble, in Fl. Brit. India 7(22): 386.1896 non Siebold \& Zucc. 1843.

Type: India: Shillong, Khasia Hills and said to have come from the Naga Hills, 1889, G.Mann s.n. (K, Iso CAL).

Vern.: U-seij naka (Khasi).

Distribution: Arunachal Pradesh, Assam, Meghalaya, Mizoram, Nagaland. [China, Myanmar].

PSEUDOSTACHYUM Munro, Trans. Linn. Soc. London 26: 141. 1868.

Type: Pseudostachyum polymorphum Munro

Pseudostachyum polymorphum Munro, Trans. Linn. Soc. London 26(1): 142. t. 4.

1868. Schizostachyum polymorphum (Munro) R.B.Majumdar in Karthik. et al., Fl. Ind. Enumerat.- Monocot. 282. 1989.

Lectotype: India: Assam, Nigrigam, January 18, 1836, Griffith 6735 (K). LT designated by Stapleton in Edinburgh J. Bot. 5l: 30. 1994.

Vern.: Bajal, Nal, Tolli (Ass.); Serim (Khasi); Wachall (Garo); Purphiok, Paphak (Lepcha); Pheling (Nepali); Talak (Myon Naga, Manipuri); Mareo, Nagachui (Tangkhul); Chal, Chail (Kuki); Rapai/ Ratai (Mara); Uanol (Bru).

Distribution: Arunachal Pradesh, Assam, Manipur, Meghalaya, Mizoram, Nagaland, Sikkim, West Bengal. [Bhutan, China, Myanmar, Nepal].

PSEUDOXYTENANTHERA Soderstr. \& R.P.Ellis, Smithsonian Contr. Bot. 72: 52. 1988.

Type: Pseudoxytenanthera monadelpha (Thwaites) Soderstr. \& R.P.Ellis (Dendrocalamus monadelphus Thwaites). 
Pseudoxytenanthera bourdillonii (Gamble) H.B.Naithani, J. Bombay Nat. Hist. Soc. 87: 440. 1990. Oxytenanthera bourdillonii Gamble, Ann. Roy. Bot. Gard. Calcutta 7: 76. 1896. Type: "Western Ghats of Travancore, grows only on steep precipitous places \& wet rocks at elevations of 3000-4000 ft.", J.F.Bourdillon s.n. (K). Vern.: Arambu (Malyali); Ponmungil (Tamil).

Distribution: Kerala. Endemic.

Pseudoxytenanthera monadelpha (Thwaites) Soderstr. \& R.P.Ellis, Smithsonian Contr. Bot. 72: 52. 1988. Dendrocalamus monadelphus Thwaites, Enum. Pl. Zeyl. 5: 376. 1864.

Lectotype: Sri Lanka: Ambagamuwa, December 1854, Thwaites C.P. 3359 (PDA). LT designated by Soderstrom \& Ellis, Smithsonian Contr. Bot. 72: 52. 1988.

Distribution: Andhra Pradesh, Karnataka, Kerala, Tamil Nadu. [Sri Lanka].

Pseudoxytenanthera stocksii (Munro) T.Q.Nguyen, Bot. Zhurn. (Moscow \& Leningrad) 76: 993.1991. Pseudoxytenanthera stocksii (Munro) H.B.Naithani, J. Bombay Nat. Hist. Soc. 87(3): 440. 1990. Dendrocalamus stocksii (Munro) M.Kumar, Remesh \& Unnikr., Sida 21(1): 95. 2004. Oxytenanthera stocksii Munro Trans. Linn. Soc. London 26(1): 130. 1868.

Type: India: Concan, Stocks s.n. (K).

Vern.: Chivari, Mes (Marathi); Konda, Oor-shema (Kannad). Distribution: Goa, Karnataka, Kerala, Maharashtra.

SAROCALAMUS Stapleton, Novon 14(3): 347. 2004.

Type: Sarocalamus racemosus (Munro) Stapleton

Sarocalamus racemosus (Munro) Stapleton, Novon 14(3): 347. 2004. Arundinaria racemosa Munro, Trans. Linn. Soc. London 26(1): 17. 1868 emend. Fargesia racemosa (Munro) T.P.Yi, J. Bamboo Res. 2: 39. 1983. Yushania racemosa (Munro) R.B.Majumdar in Karthik et. al., R.B.Majumdar in Karthik. et. al., Fl. Ind. Enumerat.- Monocot. 283.1989.

Lectotype: India: North East Himalaya [Sikkim \& Darjeeling District], Birch Hills, alt. 6000 ft., August 1857, Thomson s.n. (K). LT selected by Gamble, 1912.

Vern.: Sanu maling, Pummoon, Pat-hioo (Sikkim); Miknu, Mikner (Lepcha \& Bhutia).

Distribution: Arunachal Pradesh, Manipur, Nagaland, Sikkim, West Bengal. [Bhutan, China,

Nepal].

Sarocalamus faberi (Rendle) Stapleton, Novon 14(3): 348. 2004. Arundinaria faberi Rendle, J. Linn. Soc. 36: 435. 1904. Bashania faberi (Rendle) T.P.Yi, J. Bamboo Res. 12(2): 52.1993. Type: China: Sichuan, above Chungking, Yangtzekiang, E.Faber 1119 (Holo K).

Distribution: Arunachal Pradesh. [China].
SCHIZOSTACHYUM Nees, Fl. Bras. Enum. Pl 2(1): 535. 1829.

Type: Schizostachyum blumei Nees

Schizostachyum andamanicum M.Kumar \& Remesh, Blumea 48(1): 187-189. 2003. Schizostachyum kalpongianum M.Kumar \& Remesh, Blumea 48(1): 187-189. 2003.

Type: Saddle Peak (North Andaman) Andaman Islands, India, 25.5.2000, Remesh \& Vishwa Kumar 20780 (Holo KFRI; Iso L, MH).

Distribution: Andaman Islands. Endemic.

Schizostachyum kurzii (Munro) R.B.Majumdar in Karthik. et al., Fl. Ind. Enumerat.- Monocot. 281. 1989. Melocanna kurzii Munro, Trans. Linn. Soc. London 26(1): 134. 1868. Pseudobambusa kurzii (Munro) Ohrnb., Bamb. World Introd. ed. 4: 19. 1997. Bambusa kurzii (Munro) N.P.Balakr., Bull. Bot. Surv. India 22(1-4): 176. 1980.

Type: India: Andaman Islands, Macpherson's straits, Kurzs.n. (K).

Distribution: Andaman \& Nicobar Islands, Manipur. [Myanmar].

Schizostachyum rogersii Brandis, Indian Trees 679. 1906. Lectotype: India: Andaman Islands, Potatang Creek, February 1904, C.G.Rogers 69 (K). Lectotype designated by M.Kumar in KFRI Research Report no. 330: 79. 2009. Distribution: Andaman \&e Nicobar Islands. Endemic.

Schizostachyum seshagirianum R.B.Majumdar in Karthik. et al., Fl. Ind. Enumerat.- Monocot. 282. 1989. Stapletonia rigoense L.B. Singha, P. Niri \& R. Devi, Phytotaxa 350(1): 079 $-085.2018$.

Type: India: Arunachal Pradesh, Garsing to Eyo, Spiang, alt. 1900 ft., U.S.Rao 1794 (CAL).

Distribution: Arunachal Pradesh. Endemic.

SINARUNDINARIA Nakai, J. Jap. Bot. 11: 1. 1935; Clayton \& Renvoize, Gen. Graminum 41. 1989; Chao \& Renvoize, Kew Bull. 44(2): 365. 1989.

Type: Sinarundinaria nitida (Mitford) Nakai (Arundinaria nitida Mitford).

Sinarundinaria arunachalensis H.B.Naithani, Indian Forester 117(1): 78. 1991. Chimonocalamus longispiculatus R.B.Majumdar in Karthik. et al., Fl. Ind. Enumerat.Monocot. 276. 1989. Pleioblastus simonii sensu Naithani \& Bennet in Indian Forester 112(1): 85. 1986., non (Carr.) Nakai (1925), non Bambusa simonii Carr. (1866).

Type: India: Arunachal Pradesh, Subansiri, Bapu, Burkill 36550 (DD).

Vern.: Yen (Nishi); Hebing (Apatani).

Distribution: Arunachal Pradesh. Endemic. 
STAPLETONIA P.Singh, S.S.Dash \& P.Kumari, Nelumbo 51: 241. 2009.

Type: Stapletonia arunachalensis (H.B.Naithani) P.Singh. S.S.Dash \& P.Kumari

Stapletonia arunachalensis (H.B.Naithani) P.Singh, S.S.Dash \& P.Kumari, Nelumbo 51:241.2009. Schizostachyum arunachalensis H.B.Naithani, Indian Forester 118(3): 230. 1992. Type: India: Arunachal Pradesh, Subansiri District, Baja near Daporijo, 300 m, 17.3.1986, H.B.Naithani 1406 (Holo DD).

Vern.: Tuchur (Adi and Hill Miri).

Distribution: Arunachal Pradesh. Endemic.

TEINOSTACHYUM Munro, Trans. Linn. Soc. London 26: 142. 1868.

Type: Temostachyum griffithii Munro

Teinostachyum beddomei C.E.C.Fisch., Fl. Madras 3(9): 1860. 1931. Schizostachyum beddomei (C.E.C.Fisch.) R.B.Majumdar in Karthik. et al., Fl. Ind. Enumerat.Monocot. 281. 1989. Teinostachyum wightii Bedd., Fl. Sylv. S. India t. 323. 1873 non Munro.

Type: India: Tamil Nadu, Nilgiris, Beddome 62 (K). Lectotype designated by M.Kumar in KFRI Research Report no. 399: 128. 2011.

Distribution: Karnataka, Kerala, Tamil Nadu. Endemic.

Teinostachyum griffithii Munro, Trans. Linn. Soc. London 26(1): 143. t. 3. 1868. Schizostachyum griffithii (Munro) R.B.Majumdar in Karthik. et al., Fl. Ind. Enumerat. Monocot. 281. 1989.

Type: Burma: Prope Wulloboom "in sylvis collinis", Griffith s.n. $(\mathrm{K})$.

Distribution: Arunachal Pradesh, Assam, Meghalaya. [Myanmar].

THAMNOCALAMUS Munro, Trans. Linn. Soc. London 26(1): 33, 157. 1868.

Lectotype: Thamnocalamus spathiflorus (Trin.) Munro (Arundinaria spathiflora Trin.).

LT designated by A.Redder in Bibliogr. Cult. Trees 639. 1949.

Thamnocalamus aristatus (Gamble) E.G.Camus, Bambusees (Camus) 54. 1913. Arudinaria aristata Gamble, Ann. Roy. Bot. Gard. Calcutta 7: 18. t. 17. 1896. Thamnocalamus spathiflorus Munro, Trans. Linn. Soc. London 26(1): 34. 1868. p.p. non Trin. 1935.

Lectotype: India: Sikkim, Phalut, alt. 10000 ft., Gammie s.n. (K). LT designated by C.S. Chao \& Renvoize in Kew Bull. 44(2): 363. 1989.

Vern.: Babain, Babam (Sikkim); Rato, Nigalo (Darjeeling); Rato-nigala (Sikkim).
Distribution: Arunachal Pradesh, Assam, Manipur, Sikkim, West Bengal. [Bhutan, Nepal].

Thamnocalamus arunachalensis H.B. Naithani, in Indian Forester 141(5): 587-589. 2015.

Type: India: Arunachal Pradesh, West Siang dist., Mechuka, Segong, 1800m, , 2.9.2012, H.B.Naithani 5334 (Holo DD).

Vern.: 'Saynu' (Mompa of Mechuka).

Distribution: Arunachal Pradesh.

Thamnocalamus spathiflorus (Trin.) Munro, Trans. Linn. Soc. London 26(1): 34. 1868. Arundinaria spathiflora Trin., Mem. Acad. Imp. Sci. Saint-Petersbourg. Ser. 6, Sci. Math. Seconde Pt. Sci. Nat. 3: 617. 1835. Thamnocalamus spathiflorus Munro ssp. occidentalis Stapleton, Edinburgh J. Bot. 5 l(2): 283. 1994.

Lectotype: Nepal: North West of Himalaya, alt. $7000 \mathrm{ft}$., Numer. List [Wallich] no. 5041 (K). LT designated by C.S.Chao \& Renvoize in Kew Bull. 44(2): 363.1989.

Vern.: Ringal, Nigal (Hindi); Tham, Tham-ringal, Parikh, Deo-ringal (Garhwali).

Distribution: Arunachal Pradesh, Assam, Himachal Pradesh, Uttar Pradesh, Uttarakhand. [Bhutan, China, Nepal].

THYRSOSTACHYS Gamble, Indian Forester 20: 1. 1894.

Type: Thyrsostachys oliverii Gamble

Thyrsostachys oliveri Gamble, Indian Forester 20: 1. 1894.

Type: Burma: J.W.Oliver s.n. (K).

Vern.: Kanak-kaich (Tripura); Phunkirua (Mizo); Goping (Kuki); Burma-wa (Manipuri).

Distribution: Arunachal Pradesh, Manipur, Mizoram, Tripura, Uttarakhand, West Bengal. [China, Myanmar].

Thyrsostachys siamensis Gamble, Ann. Roy. Bot. Gard. Calcutta 7: 59-60. t. 5l. 1896. nom. cons. Thyrsostachys regia (Thomson ex Munro) Bennet, Indian Forester 114(10): 711. 1988. Bambusa regia Thomson ex Munro, Trans. Linn. Soc. London 26(1): 116. 1868.

Lectotype: Kurzs.n. (K). LT designated by Stapleton in Taxon 47: 739.1998

Distribution: Uttarakhand (Forest Research Institute, Dehra Dun), West Bengal (AJC Bose Indian Botanical Garden, Howrah). [China, Myanmar].

YUSHANIA Keng f., Acta Phytotaxonomica Sinica 6(4) 355-356. 1957.

Type: Yushania niitakaymensis (Hayata) P. C. Keng

Yushania anceps (Mitford) W.C.Lin, Bull. Taiwan Forest Res. Inst. 248: 9. 1974. Arundinaria anceps Miff., Bamb. Gard. 181. 1896. Sinarundinaria anceps (Mitf.) C.S.Chao \& Renvoize, Kew Bull. 44(2): 359. 1989. Arundinaria jaunsarensis Gamble, Ann. Roy. Bot. Gard. Calcutta 7: 23. t. 22. 1896. 
Chimonobambusa jaunsarensis (Gamble) K.N.Bahadur \& H.B.Naithani, Indian J. Forest. 1(1): 41.1978. Yushania jaunsarensis (Gamble) T.P.Yi. J. Bamboo Res. 2(1); 39. 1983. Fargesia elegans (Kurz) J.Campbell ssp. jaunsarensis (Gamble) J.Campbell, Gen. Himal. Bamb. 38. 1985.

Type: India: Uttar Pradesh, Jaunsar Hills, Mundali, alt. 7500 ft., May 1891, Gamble 23134 (K).

Vern.: Ningal, Ringal (Jaunsar); Jumra, Gyons, Sarura, Sarurha (Garhwal).

Distribution: Uttarakhand. Endemic. Cultivated in Europe and the U.S.A.

Yushania elegans (Kurz) R.B.Majumdar in Karthik. et al., Fl. Ind. Enumerat.- Monocot. 282. 1989. Arundinaria elegans Kurz, J. Asiat. Soc. Bengal Pt. 2. Nat. Hist. 42: 249. 1873. Sinobambusa elegans (Kurz) Nakai, J. Arnold Arbor. 6: 152. 1925. Fargesia elegans (Kurz) J.Campbell, Gen. Himal. Bamb. 37. 1985. Sinarundinaria elegans (Kurz) C.S.Chao \& Renvoize, Kew Bull. 44(2): 357. 1989.

Type: Burma: Martaban, Nattaung Hills, alt 5000-7000 ft., Kurzl44 (K).

Vern.: Jilli (Naga); Ngarik, Likkang (Kuki).

Distribution: Arunachal Pradesh, Nagaland. [Myanmar].

Yushania hirsuta (Munro) R.B.Majumdar in Karthik. et al., Fl. Ind. Enumerat.- Monocot. 282. 1989. Arundinaria hirsuta Munro, Trans. Linn. Soc. London 26(1): 30. 1868. Sinarundinaria hirsuta (Munro) C.S.Chao \& Renvoize, Kew Bull. 44(2): 355. 1989.

Lectotype: India: Khasi Hills, among rocks, 5600 ft., November 1835, Griffith 6726 (K). LT designated by C.S.Chao \& Renvoize in Kew Bull. 44(2): 355. 1989.

Vern.: U-stoh, U-skong, Dait-si-sai, U-stewiong (Khasi).

Distribution: Arunachal Pradesh, Assam, Meghalaya, Nagaland, Sikkim, West Bengal. [Bhutan].

Yushania maling (Gamble) R.B.Majumdar in Karthik. et al., Fl. Ind. Enumerat.- Monocot. 283. 1989. Arundinaria maling Gamble, Bull. Misc. inform. Kew 1912: 139. 1912. Fargesia maling (Gamble) J.Campbell, Gen. Himal. Bamb. 40. 1985. Sinarundinaria maling (Gamble) C.S.Chao \& Renvoize, Kew Bull. 44(2): 356. 1988.

Lectotype: India: Darjeeling District, Tonglo [Tanglu], alt. 9000 ft., May 23, 1904, Osmaston s.n. (K). LT designated by C.S.Chao \& Renvoize in Kew Bull. 44(2): 356. 1989.

Vern.: Maling bans (Sikkim, Darjeeling).

Distribution: Arunachal Pradesh, Sikkim, West Bengal. [Bhutan, Nepal].

Yushania microphylla (Munro) R.B.Majumdar in Karthik. et al., Fl. Ind. Enumerat.- Monocot. 283. 1989. Arundinaria microphylla Munro, Trans. Linn. Soc. London 26(1): 32. 1868. Bambusa microphylla Griff., J. Trav. 1: 242. 259. 1847. nom. nud.
Sinarundinaria microphylla (Munro) C.S.Chao \& Renvoize, Kew Bull. 44(2): 354. 1989.

Syntypes: Bhutan: Tashigang District, Sanah, alt. 7000 ft., Griffiths 623 (K); Bhutan-Sanah alt. 6000-10000 ft, Griffith s.n. (K).

Vern.: U-tangei (Khasi).

Distribution: Assam, Sikkim, Meghalaya. [Bhutan, Nepal].

Yushania pantlingii (Gamble) R.B.Majumdar in Karthik. et al., Fl. Ind. Enumerat.- Monocot. 283. 1989. Arundinaria pantlingii Gamble, Ann. Roy. Bot. Gard. Calcutta 7: 129. t. 118. 1896. Fargesia pantlingii (Gamble) J.Campbell, Gen. Himal. Bamb. 41. 1985. Semiarundinaria pantlingii (Gamble) Nakai, J. Arnold Arbor. 6: 15l. 1925. Sinarundinaria pantlingii (Gamble) C.S.Chao \& Renvoize, Kew Bull. 44(2): 359. 1989.

Type: India: Darjeeling District, Sikkim border "Hills of British Bhutan", Rechi La 11000 ft., September 1895, Pantling's collectors s.n. $(\mathrm{K})$.

Vern.: Nigale, Maling (Sikkim).

Distribution: Arunachal Pradesh, Sikkim, West Bengal. [Bhutan, Nepal].

Yushania rolloana (Gamble) T.P.Yi, J. Bamboo Res. 2(1): 39. 1983. Arundinaria rolloana Gamble, Ann. Roy. Bot. Gard. Calcutta 7: 24. t. 23. 1896. Sinarundinaria rolloana (Gamble) C.S.Chao \& Renvoize, Kew Bull. 44(2): 555. 1989.

Type: India: Assam, Naga Hills, Zullah Valley, alt. 5000-7000 ft., 1889, Gopal Baneriee- J.

Rollo s.n. (K).

Vern.: Jipvo (Naga).

Distribution: Assam, Nagaland. Endemic.

Yushania yadongensis T.P.Yi, J. Bamboo Res. 4(2): 33. 1985. Yushania longissimi K.F.Huang \& Q.F.Zheng, Wuyi Science Journal 2(2): 20, f. 3. 1982.

Type: n.v.

Distribution: Sikkim. [Bhutan].

\section{CULTIVATED SPECIES}

Bambusa affinis Munro, Trans. Linn. Soc. London 26(1): 93. 1868.

Type: Hab. in Ind. or. Burmah, Yoongalum (Teeshe incolis), Brandis 18 (HBG).

Distribution: West Bengal. [Myanmar].

Bambusa copelandii Gamble, Indian Trees 671. 1906. Sinocalamus copelandii (Gamble) Raizada, Indian Forester 74(1): 10. 1948.

Lectotype: India: Dehra Dun, cultivated, alt. 2200 ft; September. 1898; Gamble 27166 (K). LT designated by Xia \& Stapleton in Kew Bull. 52: 484. 1997. 
Distribution: Kerala (Tropical Botanical Garden and Research Institute), Uttarakhand (Forest Research Institute, Dehra Dun). [Myanmar].

Bambusa wamin E.G.Camus, Bambusees (Camus) 135. 1913. Bambusa vulgaris var 'wamin' Brandis ex McClure, 162. 1966. Type: "Cultivated in the garden of Northern Shan States of China. Brought from china, by others from Chengmai, Siam (Troupe; May 1902)."

Vern.: U-siej-jympun (Khasi); Wasshuti (Garo).

Distribution: India [China, Thailand]. Widely cultivated in gardens and landscaping.

Dendrocalamus asper (Schult. \& Schult.f.) Backer ex Heyne, Nutt. Pl. Ned.-Ind., ed. 2, 1:301. 1927. Bambusa aspera Schult. \& Schult. f., Syst. Veg., ed. 15 bis [Roemer \& Schultes] 7(2): 1352-1353.1830.

Type: "Arundarbor aspera Rumphius, Herb. Ainb. 4:11. In Amboina et Huamchela ad montium pedes."

Distribution: Kerala, Uttarakhand, West Bengal. [China, Myanmar]. Cultivated mainly for the edible young shoots.

Gigantochloa atroviolacea Widjaja, Reinwardtia 10(3): 323. 1987.

Type: Indonesia: Java, Wonosari, Nagasari, February 1983, Ramlanto s.n. (BO).

Distribution: West Bengal. [Malaysia].

Gigantochloa pseudoarundinacea (Steud.) Widjaja, Reinwardtia 10(3): 305. 1987. Bambusa pseudoarundinacea Steud., Syn. Pl. Glumac. I: 330. 1854. Bambusa nagalandiana H.B.Naithani, Indian Forester 133(9): 1267-1269. 2007.

Type: Indonesia: Java, H.Zollinger 3479 (L).

Vern.: Ralleng mau (Mizo).

Distribution: Meghalaya, Mizoram, Nagaland.

Neololeba atra (Lindl.) Widjaja, Reinwardtia 11(2): 114. 1997. Bambusa atra Lindl., Penny. Cyclop. 3: 357. 1835. Bambusa lineata Munro, Trans. Linn. Soc. London 26(1): 118. 1868.

Type: found in Amboyna.

Distribution: West Bengal (AJB Bose Indian Botanic Garden, Howrah). Its presence in Andaman Islands is recorded only based on literature and no specimen is available in any herbaria from these islands. [New Guinea, the Moluccas, Sangihe Island, Philippines].

Phyllostachys aurea (Andre) Riviere. \& C. Riviere, Bull. Soc. Acclim. France, ser. 3, 5: 716. f. 36. 37. 1878. Bambusa aurea Andre, Pl. Feuill. Ornem.: 102. 1866.

Type: Tunis. Cultivated in "Jardim du Hamma", Anonymous s.n. (P).

Distribution: Himachal Pradesh (Shimla, Kasauli). [China].
Phyllostachys nigra (Lodd. ex Lindl.) Munro, Trans. Linn. Soc. London 26(1): 38, 23.1868. Bambusa nigra Lodd. ex Lindl., Penny Cyclop. 3: 357. 1835.

Type: "China, Japonia; Siebold, Burger, Textor; ins. Niphon Osakka in oryzetis, Pierot. in hortis Europae, Chinae et Indiae culta."

Vern.: Seij-naka-iong (Khasi).

Distribution: India. Frequently used as an ornamental plant in gardens and nurseries. Native to S. Hunan, China.

Pseudosasa japonica Makino ex Nakai, J. Arnold Arbor. 6: 150. 1925. Arundinaria japonica Siebold \& Zucc. ex Steud., Syn. Pl. Glumac. 1: 334. 1854.

Type: "Java" Japan, Metake, P.F. von Siebold s.n. (L).

Distribution: Meghalaya (Ward's Lake Garden, Shillong), West Bengal (AJC Bose Indian Botanic Garden, Howrah). [China].

Pseudoxytenanthera parvifolia (Brandis ex Gamble) T.Q.Nyngen, Bot. Zurn. (Moscow and Leningrad) 76(7): 993 1991. Oxytenanthera parvifolia Brandis ex Gamble, Ann. Roy. Bot. Gard. Calcutta 7: 72. t. 63. 1896. Gigantochloa parvifolia (Brandis ex Gamble) T.Q.Nguyen, Bot. Zhurn. 75(2): 224. 1990.

Type: Burma: Yonzalin Valley, March 1880, D.Brandis s.n. (HBG).

Distribution: Assam, Mizoram. [Myanmar].

Schizostachyum brachycladum (Kurz ex Munro) Kurz, J. Asiat. Soc. Bengal Pt. 2. Nat.. Hist.39 (2): 89. t. 6. f. 2. 29. 1870. Melocanna Zollinger (Steud.) Kurz ex Munro var. brachyclada Kurz ex Munro, Trans. Linn. Soc. London 26(1): I 34. 1868. Schizostachyum zoliingeri Steud., Syn. Pl. Glumac. 1: 332. 1854. Type: Java

Distribution: Keral (TBGRI). [Indonesia, Malayasia, Philippines, Singapore].

Shibataea kumasasa (Zoll. ex Steud.) Makino, Bot. mag. Tokyo 28: 22. 1914. Bambusa kumasaca Zoll. ex Steud., Syn. Pl. Glumac. 1: 331. 1854. Phyllostachys kumasasa (Zoll. ex Steud.) Munro, Trans. Linn. Soc. London 26: 39. 1868.

Distribution: Keral (TBGRI), Manipur (KVK, Hengbung, Senapati dist.), West Bengal (AJC Bose Indian Botanic Garden, Howrah; Llyodd Botanic Garden, Darjeeling). [China, Japan].

Yushania niitakayamensis (Hayata) Keng f., Acta Phytotax. Sin. 6: 357. 1957. Arundinaria niitakayamensis Hayata, Bot. Mag Tokyo 21: 49. 1907. Sasa niitakayamensis E.G.camus, Bambusees 24. 1913.

Distribution: Meghalaya (Ward's Lake Garden, Shillong). [China, Philippines, Taiwan]. 


\section{SPECIES OF DOUBTFUL OCCURRENCE}

Arundinaria gracilis (Riviere \& C.Riviere) E.G.Camus, Rev. Hort. [Paris]. 58: 490. 1886. Bambusa gracilis Riviere \& C. Riviere. Bull. Soc. Natl. Acclim. France Ser. 3. 5(25): 682. 1878.

Type: No type cited.

Distribution: Arunachal Pradesh, Sikkim, West Bengal. Endemic?

Bambusa griffithiana Munro, Trans. Linn. Soc. London 26(1): 99. 1868.

Type: "Hab. in Ind. or. Burmah, Mogoung, Griffith s.n".

Distribution: Manipur. [Myanmar].

Bambusa kingiana Gamble, Ann. Roy. Bot. Gard. Calcutta 7: 46. t. 421896.

Type: Burma: Kama District, Petsut, J.W.Oliver s.n.

Distribution: Manipur. [Myanmar].

Bambusa longispiculata Gamble ex Brandis, Indian Trees 668. 1906.

Type: Bangladesh: Chittagong Hill tracts, Ruby mine District, alt. 3500 ft., Griffith s.n.

Distribution: Meghalaya, Mizoram, Tamil Nadu, Uttar Pradesh, West Bengal. [Bangladesh, China, Myanmar].

Cephalostachyum flavescens Kurz, J. Asiat. Soc. Bengal Pt 2 Nat. Hist. 42: 252. 1873.

Type: Pegu: H.B.C. culta.

Schizostachyum flavescens (Kurz) R.B.Majumdar in Karthik. et al., Fl. Ind. Enumerat.- Monocot. 281. 1989.

Distribution: Andaman \& Nicobar Islands, Tamil Nadu. [Myanmar].

Chimonobambusa armata (Gamble) J. R. Xue \& T. P. Yi, J. Bamboo Res. 2(1): 38.

1983. Arundinaria armata Gamble Ann. Roy. Bot. Card. Calcutta 7: 130. t. 119. 1896. Chimonocalamus armatus (Gamble) R.B.Majumdar in Karthik. et al., Fl. Ind. Enumerat.Monocot. 275. 1989. Oreocalamus armatus (Gamble) T.H.Wen, J. Bamboo Res. 5(2): 22.1986.

Type: Burma: "Hills of Upper Burma, Bernardmyo", J.W.Oliver 1894 (K).

Distribution: India. [China, Myanmar].

Dendrocalamus collettianus Gamble, Ann. Roy. Bot. Gard. Calcutta 7: 93. 1896.

Type: upper Myanmar, Fort Stedman Abul Huck s.n. (Isotype CAL).

Distribution: West Bengal (AJC Bose Indian Botanic Garden, Howrah). [Myanmar].

Dinochloa scandens (Blume) Kuntze, Revis. Gen. Pl. 773. 1891. Bambusa scandens Blume, Flora 7: 291. 1824.
Type: Java: Megamendung, Blume s.n. (L).

Distribution: Andaman $\&$ Nicobar Islands. [Indonesia].

Gigantochloa atter (Hassk.) Kurz, Natuurk. Tijdschr. Ned. Indie 27: 226. 1864.

Syntypes: Indonesia: Java, Horsfield s.n.; Java: Buitenzorg, Kurz s.n.

Distribution: West Bengal. [Malaysia].

Gigantochloa macrostachya Kurz, J. Asiat. Soc. Bengal. Pt. 2, Nat. Hist. 42: 251. 1873. Gigantochloa tekserah Camus, Bamb. 141. 1913.

Type: Martaban, Tenasserim.

Distribution: Arunachal Pradesh, Assam, Meghalaya, Mizoram. [Bangladesh, Myanmar].

Gigantochloa nigrociliata (Buse) Kurz, Natuurk. Tijdschr. Ned.-Indie 27: 226. 1864 var. nigrociliata. Bambusa nigrociliata Buse, Pl. Jungh. 389. 1854. Oxytenanthera nigrociliata (Buse) Munro, Trans. Linn. Soc. London 26(1): 228. 1868.

Type: "Habitat insulatn Javae Prope Tjiberem", Junghuhn s.n.

Distribution: Andaman \& Nicobar Islands, Kerala. [Myanmar, Indonesia].

Gigantochloa rostrata K.M.Wong, Malaysian Forester 45(3): 349. 1982.

Type: Malaya: October 15, 1980, KM.Wong KEP FRI28981 (L). Vern.: Wa-shut (Garo).

Distribution: Andaman \& Nicobar Islands, Assam, Bihar, Karnataka, Madhya Pradesh, Maharashtra, Meghalaya, Orissa, Tripura. [Bangladesh].

Kuruna debilis (Thwaites) Attigala, Kathr. \& L.G.Clark, Phytotaxa 174(4): 200. 2014. Arundinaria debilis Thwaites, Enum. Pl. Zeyl. [Thwaites] 375. 1864. Type: "Hab. Elevated parts of the Central Province". Ceylon, CP 1 Thwaites (K).

Distribution: Manipur. [Sri Lanka]

Pleioblastus simonii (Carriere) Nakai, J. Arnold Arbor. 6(3): 147. 1925. Bambusa simonii Carriere, Rev. Hort. [Paris]. 37: 380.1866.

Type: China?: 1862, E.Simon s.n. (P).

Distribution: Arunachal Pradesh. [Japan].

\section{RESULTS AND DISCUSSION}

Present work based on extensive taxonomic research on this group of plants for more than fifteen years from 2002 to 2018 , enumerates total of 148 species and 6 varieties under 33 genera from India including indigenous, widely cultivated and naturalized and the exotic species used particularly for ornamental purposes in landscaping gardens. It also includes the species mentioned as occurring in India in previous works but found of doubtful occurrence in the present work. 
Three new combinations have been proposed according to the recent generic treatment and the morphological characters of the plants.

\section{ACKNOWLEDGEMENTS}

Author is thankful to the Ministry of Environment, Forest and Climate Change, Govt. of India, New Delhi and the Director Botanical Survey of India for every help and support to carry the reseach. Forest officials of different states are also acknowledged for their help during the field explorations. All the reseachers and the staffs of Botanical Survey of India are also duly acknowledged for being helpful in different ways during the work.

\section{REFERENCES}

Bennet, S.S.R. \& Gaur, R.C. (1990). Thirty seven bamboos growing in India. Forest Research Institute, Dehra Dun.

Bentham, G. (1881). Notes on Gramineae. J. Linn. Soc. Bot. 19: 14-134.

Blatter, E. (1929). Indian bamboos brought up-to-date. Indian Forester 55 541-562; 586-612.

Brandis, D. (1906). Indian Trees. Archibald Constable \& Co. Ltd., London.

Camus, E. G. (1913). Les Bambusees: monographic, biologic. Culture. Principaux usages. Paul Lechevalier, Paris. https://doi.org/10.5962/bhl.title.15463

Dash S. S., P. Kumari \& Singh P. (2009). Notes on flowering in Schizostachyum arunachalensis Naithani (Poaceae: Bambusoideae) NELUMBO 51.

Dransfield, S. \& Widjaja, E.A. [Eds.] (1995). Plant Resources of South East Asia No. 7. Bamboos. Bachyuys Publishers, Leiden.

Holttum, R. E. (1958). Bamboos of the Malay Peninsula. Gard. Bull. Singapore 16: 1-135.

Kunth, C. S. (1815). Considerations generales sur les Graminees, Memoires du Museum d' Histoire Naturelle, 2: 62-75.

Kurz, S. (1877). Forest Flora of British Burma 2 vols. Govt. Printing, Calcutta.

Linnaeus, C. (1753). Species Plantarum (Ed. 1), Vol. 2. Stockholm.

Majumdar, R. B. (1989). Bambusoideae. In S. Karthikeyan, S.K. Jain, M.P. Nayar \& M. Sanjappa (Eds.) Florae Indicae Enumeratio - Monocotyledonae. Botanical Survey of India, Calcutta, pp. 274-254. https://doi.org/10.1111/j.1756-1051.1998.tb01868.x

Munro, W. (1868). A Monograph of the Bambusaceae, including descriptions of all the species. Trans. Linn.Soc. London 26 (1): 1-157, tt. 1-6. https://doi.org/10.5962/bhl.title.15634

Nees, von Esenbeck, C. G. D. (1835). Bambuseae brasilienses: recensuit et alias in India Orientalis provenientes adjecit, Linnaea 9: 461-494.

Negi, S.S. \& Naithani H.B., (1994). Handbook of Indian Bamboos. Oriental Enterprises, Dehra Dun, pp. 1-234.
Parker, R. N. (1929). The Indian bamboos brought up-to-date (Additional Notes). Indian Forester 55: 612-613.

Retzius, A.J. 1789 (1788). Observationes Botanicae....... quibus accedunt Joannis Gerhardi Koeing. Vol. 5. Leipzig.

Rheede tot Draakestein, H. A. Von (1678-1703). Hortus Indicus Malabaricus, Amsterdam.

Roxburgh, W. (1814). Hortus Benghalensis, A Catalogue of the Plants growing in The Honourable East India Company's Botanic Garden at Calcutta. Mission Press, Serampore.

Ruprecht, F. J. (1839). Bambuseae monographice exponit. Typis Academiae Caesareae Scientiarum, St. Petersburg.

Schreber, J. C. D. (1789). Caroli a Linne Genera Plantarum - Edito Octava post Reichardianum secunda prioribus longe auctior atque emendatior, l; Frankfurt.

Stapleton C. M. A (2000). Bambuseae in Noltie, H.J., Flora of Bhutan 3(2) 482-514. Royal Botanic Garden Edinburgh, Royal Govt. of Bhutan.

Tiwari, D.N. (1992). A Monograph on Bamboo. International Book Distributors, Dehra Dun.

Vorontsova, S. Maria, Clark, G. Lynn, John Dransfield, Rafael Govaerts and Baker, J. William (2016). World Checklist of Bamboos and Rattans. INBAR technical report no. 37.

( 2019 | Published by Plantae Scientia 